\title{
Dijital Eğitsel Oyun Geliştirme Öz-Yeterlik Ölçeğinin Geliştirilmesi
}

\author{
Özge KELLECI* Taibe KULAKSIZ ${ }^{* *}$
}

• Geliş Tarihi:8.04.2020 • Kabul Tarihi: 01.09.2020 • Çevrimiçi Yayın Tarihi: 06.11.2020

\section{$\ddot{\mathbf{O} z}$}

Bu çalışmanın amacı geçerli ve güvenilir bir Dijital Eğitsel Oyun Geliştirme Öz-yeterlik Ölçeği (DEOGÖÖ) geliştirmektir. Bu bağlamda ilk olarak alanyazın taraması yapılmış ve madde havuzu oluşturulmuştur. Sonrasında, uzman görüşlerine göre her bir ölçek maddesi göz önünde bulundurularak kapsam geçerlik oranı hesaplanmıştır. 24 madde içeren ilk taslak ölçek, 418 kişiye uygulanarak geçerlik ve güvenirlik çalışmaları yürütülmüştür. Veriler SPSS programı kullanılarak analiz edilmiştir. DEOGÖÖ'nün yapı geçerliğini test etmek amacıyla Açımlayıcı Faktör Analizi (AFA) ve Doğrulayıcı Faktör Analizi (DFA) yapılmıştır. AFA'ya uygunluğu test etmek için KaiserMeyer-Olkin (KMO) katsayısı .965 olarak hesaplanmış ve Bartlett Küresellik Testi p =.000 düzeyinde anlamlı bulunmuştur. AFA analizi sonucunda 21 maddeden oluşan ölçeğin Eğitim ve Oyun geliştirme şeklinde tanımlanan iki faktörlü bir yapıya sahip olduğu ve bu faktörlerin toplam varyansın \%70.25'ini açıkladığı saptanmıştır. Ölçek maddelerinin ayırt edicilik düzeylerini belirlemek amacıyla yapılan madde analizi sonucunda, maddeler arasındaki farkın .01 düzeyinde anlamlı olduğu ortaya çıkmıştır. Son olarak ölçek için Cronbach alfa güvenirlik katsayısı .972 olarak hesaplanmıştır. DFA sonucunda, model kabul edilebilir düzeydedir.

Anahtar sözcükler: dijital eğitsel oyun, ölçek geliştirme, öz-yeterlik, dijital eğitsel oyun geliştirme öz-yeterliği, yükseköğretim

\section{Atıf:}

Kelleci, Ö., Kulaksız, T. (2021). Dijital eğitsel oyun geliştirme öz-yeterlik ölçeğinin geliştirilmesi. Pamukkale Üniversitesi Eğitim Fakültesi Dergisi, 52, 1-30.doi:10.9779/pauefd.716426.

\footnotetext{
* Dr. Öğr. Üyesi, Hasan Kalyoncu Üniversitesi, Eğitim Fakültesi, Eğitim Programları ve Öğretim Bölümü, ozge.kelleci@hku.edu.tr, ORCID: 0000-0001-6495-8320

** Dr. Sağlık Bilimleri Üniversitesi, taibekulaksiz@gmail.com, ORCID: 0000-0002-7960-7440
} 


\section{Giriș}

Yirmi birinci yüzyılda gerçekleşen teknolojik gelişmeler toplumlarda sosyo-ekonomik, fiziksel ve psikolojik pek çok değişime yol açmıştır. Bu dijital dönüşüm, eğitimde de görülmektedir. Bu yeni toplumun ihtiyaçlarını karşılamak amacıyla eğitimde yeni yöntem ve yaklaşımlar kullanılmaya başlanmıştır. Bunun yanı sıra öğrenme ortamları farklılaşmış, eğitim teknolojileri ile zenginleştirilmiş öğrenme materyallerinin kullanımı daha çok önem kazanmıştır. Eğitimde kullanılan yeni yöntem ve yaklaşımlar arasında eğitimde başarıyı ve motivasyonu arttırmak için kullanılabilecek etkili yöntemlerden biri de dijital oyun tabanlı öğrenme yaklaşımıdır (Brom, Dechterenko, Sisler, Hlavka ve Lukavsky, 2018; Byun ve Joung, 2018; Chen, Shih ve Law, 2020; Tüzün, Barab ve Thomas, 2019; Yang, 2015; Yien, Hung, Hwang ve Lin, 2011). Dijital oyun tabanlı öğrenme yaklaşımında, kişiselleştirilebilir ve öğrenme ihtiyaçlarına uygun olarak tasarlanan Dijital Eğitsel Oyunlar (DEO) aracılığı ile öğrenme daha etkili bir şekilde gerçekleşmektedir (Li, Lemieux, Vandermeiden ve Nathoo, 2013; Prensky, 2001; Talan, Doğan ve Batd1, 2020).

1970'den bu yana bireylere akademik becerileri kazandırmak için kullanılan DEOlar (Noemi ve Maximo, 2014), soyut ve kavramsal bilgilerin kazandırılmasında, psikomotor ve üst bilişsel becerilerin ediniminde de etkili olmaktadır (Law ve Sun, 2012). DEOlar bireylerin yaparak öğrenmesine ve bilginin gerçek yaşamda uygulanabilir şekilde transfer edilebilmesine odaklanmıştır (An ve Cao, 2017). DEO'ların eğitimde etkili olabilmesi için öğrencilerin öğrenme ihtiyaçlarına, DEO geliştirme modellerine ve standartlarına göre geliştirilmesi önemlidir (Li ve diğerleri, 2013). Öğretmenler, programcı/mühendisler veya öğretmen adayları/öğrenciler gibi potansiyel dijital eğitici oyun geliştiricilerin de dijital eğitsel oyun geliştirme yeterliklerine sahip olması önemlidir. Nitekim 21. yüzyıl eğitim becerilerinde potansiyel dijital eğitsel oyun geliştiricilerden biri olan öğretmenlerin genel yeterliklerinden birinin de dijital öğrenme araçlarını ve materyallerini öğrencilerin ihtiyaçlarına yönelik ve etkili bir şekilde geliştirebilmeleri olduğu ifade edilmektedir. (International Society for Technology in Education [ISTE], 2017; Milli Eğitim Bakanlığı (MEB), 2020).

Araştırmalar DEOların eğitime olan katkılarını açıkça ortaya koymaktadır. Ancak öğretmenlerin DEO geliştirme ve DEOları eğitim programına entegre etme becerilerinin düşük olması, DEOların eğitimde etkili bir araç olarak kullanılmasını engelleyebilmektedir (An, Haynes, D'Alba ve Chumney, 2016; Gaudelli ve Talyor 2011; Gros, 2015; Hava ve 
Cakir, 2017; Li ve diğerleri, 2013; Prensky, 2008; Sancar Tokmak ve Özgelen, 2013). Sancar Tokmak ve Özgelen (2013) tarafından yapılan çalışmada, her dijital eğitsel oyunun öğrencilerin öğrenme ihtiyaçlarını karşılamadığı ve öğretmenlerin de eğitim içeriğine uygun dijital eğitsel oyunu seçerken zorlandıkları ifade edilmiştir. Martins ve Oliveira (2018) tarafından yapılan ve öğretmenlere dijital oyun tasarlama eğitiminin verildiği çalışmada, dijital oyun geliştirme becerileri yüksek olan öğretmenlerin, öğrencilerinin de motivasyon ve öğrenme düzeylerinin yüksek olduğu vurgulanmıştır. Yine aynı çalışmada öğretmenlerin dijital eğitsel oyun geliştirme yeterliklerinin düşük olduğu da ifade edilmiştir. Hava ve Cakir (2017) çalışmalarında dijital eğitsel oyun geliştirmenin öğrencilerin 21. yüzyıl becerilerinden, problem çözme, yaratıcı düşünme ve programlama becerilerini geliştirdiğini ortaya koymuş ve dijital oyun geliştirme eğitimindeki en büyük engelin, öğretmenlerin dijital eğitsel oyun geliştirmeye yönelik deneyim eksiklikleri ve yetersizlikler olduğuna dikkat çekmiştir.

Diğer yandan bir bireyin bir işi yapabilme yeterliği, doğrudan o işi başarabilme yeterliği konusundaki yargısını da etkilemektedir. Bireyin belirli bir performansı göstermek için gerekli etkinlikleri düzenleyip başarılı bir şekilde yapma kapasitesine ilişkin yargısı sosyal öğrenme kuramına göre öz-yeterlik olarak tanımlanmaktadır (Bandura, 1997). Bireyin belli bir alana yönelik öz-yeterlik algısının yüksek olması, o alana ilişkin etkinliklere katılmakta daha istekli olmalarını ve o alanda karşılaştıkları problemlerle daha kolay başa çıkabilmelerini sağlamaktadır (Cherian ve Jacob, 2013). Bir bireyin öz yeterliği her durumda aynı düzeyde olmayabilir. Öğretmenlerin durum, konu veya işe bağlı olarak farklılık gösteren öz-yeterlik düzeylerinin, ilgili konu bazındaki değişkenleri etkilediği unutulmamalı ve buna göre değerlendirme yapılmalıdır. Buradan yola çıkarak dijital eğitsel oyun geliştirmeye yönelik öz yeterliği yüksek olan öğretmenlerin öğrenci ihtiyaçlarına yönelik daha etkili dijital eğitsel oyunlar tasarlayabilme yeterliğine sahip olacakları da söylenebilir. $\mathrm{Bu}$ nedenle öğretmenlere veya diğer potansiyel DEO geliştiricilere dijital eğitsel oyun geliştirme eğitimi vermek önem arz etmektedir. Bu bağlamda, bir öğretim tasarım modeli çerçevesinde DEO geliştiricilerin pedagoji, teknik ve tasarım becerilerini geliştirecek eğitimler verilmektedir (Brown, Lee ve Alejandre, 2009). Dijital eğitsel oyun geliştirme süreçlerinde yararlanılan öğretim tasarım modelleri incelendiğinde, bu sürecin genel olarak analiz, tasarım, geliştirme, uygulama ve değerlendirme aşamalarını kapsadığı görülmektedir (Akgün, Nuhoğlu, Tüzün, Kaya ve Çınar, 2011; Akıllı ve Çăğltay, 2006; Cai, Liu ve Liang, 2010; Kelleci ve Kulaksız, 2018b; Kocadere, Özhan, Bayrak ve Kibar, 2019; McMahon, 
2009; Zin, Jaafar ve Yue, 2009). Oyun geliştirmeye yönelik yeterliklerin belirlendiği çalışmalar da mevcuttur (Aleem, Capretz ve Ahmed, 2016; Hewner ve Guzdial, 2010; McGill, 2008). Bu çalışmalarda oyun geliştirme süreci ve oyun geliştiricilerin sahip olması gereken yeterlikler olmak üzere iki kriter dikkate alınmış ve her ikisi de tartışılmıştır. Bu yeterliklerin genel olarak teknik (programlama), tasarım ve yönetim boyutlarında olduğu görülmektedir.

Ayrıca DEOların etkili biçimde geliştirilebilmesi için potansiyel djital eğitsel oyun geliştiricilerin de yeterliklerinin belirlenmesi ve artırılmasına yönelik çalışmalar yapılması önemlidir. Dijital eğitici oyun geliştirme eğitimlerinde DEO geliştirme becerilerinin ve özyeterlik düzeylerinin artırılması amaçlanmaktadır. Bu nedenle, potansiyel dijital eğitsel oyun geliştiricilerin DEO geliştirme öz-yeterlik düzeylerinin belirlenmesi önemlidir. Ancak literatür incelendiğinde dijital oyun geliştirme öz-yeterlik ölçeğine rastlanmamıştır. Bu alanda, Eğitim Yazılımı Geliştirme Öz-yeterlik Ölçeği (Aşkar ve Dönmez, 2004), Öğretim Materyali Tasarlama Öz-Yeterlik Öçeği (Bakaç ve Özen, 2015), Bilgisayar Programlama Öz-Yeterlik Ölçeği (Ramalingam ve Wiedenbeck, 1998) mevcuttur. Ancak bu ölçekler spesifik olarak dijital oyun geliştirme öz-yeterlik düzeyini ölçmemektedirler. $\mathrm{Bu}$ gereksinimlerle, bu araştırmanın ana amacı geçerli ve güvenilir bir Dijital Eğitsel Oyun Geliştirme Öz-yeterlik Ölçeği (DEOGÖÖ) geliştirmektir. Ayrıca, oyun geliştiricilerin DEO geliştirme kursu alma durumları ve DEO geliştirme deneyimlerine göre dijital eğitsel oyun geliştirme öz-yeterlik düzeylerini belirlemek de amaçlanmıştır.

\section{Yöntem}

\section{Katılımcilar}

$\mathrm{Bu}$ çalışma kapsamında geliştirilen DEOGÖ ölçeği potansiyel dijital eğitsel oyun geliştiricileri içindir. Buna göre, araştırmanın ilgili katılımcılar çerçevesinde gerçekleştirilebilmesi için Hasan Kalyoncu Üniversitesi Sosyal Bilimler Enstitüsü Etik Kurulu tarafından 2003160012 sayılı etik kurul onayı alınmıştır. Araştırmaya katılımda gönüllülük esas alınmıştır. Bu doğrultuda katılımcılar çalışmaya amaçlı örneklem yöntemine göre dahil edilmiştir. Araştırmanın amacı doğrultusunda belirli özelliklere sahip katılımcılar seçildiğinde amaçlı örneklem tercih edilmektedir (Büyüköztürk, Çakmak, Akgün, Karadeniz ve Demirel, 2015). Katılımcılar, Bilgisayar ve Öğretim Teknolojileri Eğitimi (BÖTE), Sınıf Öğretmenliği ve Bilgisayar Mühendisliği bölümü öğrencileri ile mezunlarından oluşmaktadır. Bu bağlamda katılımcılar, dijital eğitsel oyun geliştirme konusunda eğitim 
almış, gerekli önkoşul becerilere sahip olan veya bu alanda çalışanlardır. Buna göre 20182019 eğitim-öğretim yılında amaçlı örnekleme yöntemine göre belirlenen 418 katılımcıdan veri toplanmıştır. AFA, yaşları 18 ile 43 arasında değişen 218 katılımcı ile gerçekleştirilmiştir. DFA ise yaşları 18 ile 41 arasında değişen 200 katılımcı ile yapılmıştır. Katılımcıların demografik bilgileri Tablo 1'de sunulmuştur. Ölçek geliştirme çalışmalarında örneklem sayısının madde sayısının en az beş katı olması yeterlidir (Bryman ve Cramer, 1999; Tavşanc1l, 2002). Bu çalışmada taslak ölçek maddelerinin madde sayısı 24 olduğu için katılımcı sayısının uygun olduğu söylenebilir.

Tablo 1. Katılımcıların demografik bilgileri

\begin{tabular}{|c|c|c|c|c|c|}
\hline & & \multicolumn{2}{|c|}{ AFA } & \multicolumn{2}{|c|}{ DFA } \\
\hline & & $\mathrm{N}$ & $\%$ & $\mathrm{~N}$ & $\%$ \\
\hline \multirow{3}{*}{ Cinsiyet } & Kadın & 101 & 46.33 & 118 & 59 \\
\hline & & & & & \\
\hline & Erkek & 117 & 53.67 & 82 & 41 \\
\hline \multirow{5}{*}{ Eğitim Düzeyi } & 1. sinif & 23 & 10.55 & 5 & 2.5 \\
\hline & 2. $\sin 1 f$ & 48 & 22.02 & 37 & 18.5 \\
\hline & 3. $\sin 1 f$ & 29 & 13.30 & 104 & 52 \\
\hline & 4. $\sin 1 f$ & 58 & 26.61 & 29 & 14.5 \\
\hline & Mezun & 60 & 27.52 & 25 & 12.5 \\
\hline Toplam & & 218 & 100 & 200 & 100 \\
\hline
\end{tabular}

\section{Ölçeğin Geliştirilmesi}

Bir ölçme aracının geliştirilmesinde izlenmesi gereken adımlar genel olarak ölçme aracıyla neyin ölçülmek istediğine karar verme, maddeleri yazma, uzman görüşleri alma, uygulama, geçerlik ve güvenirlik çalışmaları yapma şeklindedir (Büyüköztürk ve diğerleri, 2015; DeVellis, 2003; Worthington ve Whittaker, 2006). DEOGÖÖ’nin geliştirme sürecinin adımları Şekil 1'de özetlenmiştir. Bu kapsamda öncelikle ölçek geliştirilirken DEOGÖÖ ile ne ölçülmek istendiğine karar verilmiş ve veri toplama aracının türü belirlenmiştir. 
İkinci olarak literatür taramasına, DEO geliştirmek için kullanılan ilgili modellere ve araçlara dayalı olarak 21 maddelik madde havuzu oluşturulmuştur. Veri toplama aracinın türünün 5'li Likert tipi bir ölçek olmasına karar verilmiştir. Bunun nedeni, katılımcıların inanç, tutum, düşünce ve davranış gibi ölçülecek değişkenlere yönelik oluşturulan maddelere katılım derecelerini belirlemek için Likert tipi ölçeklerin kullanılmasıdır (DeVellis, 2003; Gürbüz ve Şahin, 2018). Ölçekte yer alan maddelere verilen yanıtlar "Tamamen Katılmıyorum” (1) ve “Tamamen Katılıyorum” (5) arasında değişmektedir. 21 maddelik 5'li Likert taslak form oluşturulmuştur.

Üçüncü adım olarak uzmanların görüşleri alınmıştır. Buna göre taslak form, BÖTE alanında minimum yüksek lisans derecesine sahip 17 alan uzmanına içerik olarak değerlendirilmek üzere sunulmuştur. $\mathrm{Bu}$ uzmanlar, DEO geliştirmek için gerekli bilgi ve becerilere ve ölçek geliştirme deneyimine sahiptir. Taslak formda yer alan maddelerle ilgili uzman görüşlerinin alınması için 3'lü Likert yanıt türüne dayalı (Gerekli, Gereksiz ve Geliştirilmeli) bir form hazırlanmıştır. Ayrıca uzmanlardan konuyla ilgili görüş ve önerilerini formda belirtmeleri istenmiştir. Görüşlerin ardından revize edilen DEOGÖÖ taslak formu, dil-anlam açısından değerlendirilmek üzere 1 Türk dili uzmanının görüşüne sunulmuştur. Ardından alan uzmanlarından alınan değerlendirmelere göre maddelerin Kapsam Geçerlilik Oranı (KGO) hesaplanmıştır. Buna göre maddelerin KGO’su .33 ile .86 arasında değişmektedir. KGO’nun anlamlılı̆̆ını test etmek için Veneziano ve Hooper (1997) tarafindan $\alpha=.05$ anlamlılık düzeyine göre bir tablo oluşturulmuştur. Bu tabloya göre her bir maddenin ölçeğe dahil edilebilmesi için KGO'nun minimum .49 olması gerekmektedir. Elde edilen verilerde .49'un altında olan 1 madde $(\mathrm{KGO}=.33)$ formdan çıkarılmıştır. Uzmanlardan alınan görüş ve önerilere göre taslak forma 4 madde eklenmiştir. Dil-anlam ve içerik açısından gerekli revizyonlar yapılmıştır. Sonuç olarak, 24 maddelik DEOGÖÖ taslağı oluşturulmuştur.

Uygulama aşamasında 24 maddelik taslak DEOGÖÖ, ek bir yönerge metni ile Google Formlar aracılığıyla çevrimiçi olarak hazırlanmıştır. Ardından taslak ölçek formu ilgili sosyal medya grupları ve sayfalarında paylaşılmıştır. Taslak ölçek 438 katılımcı tarafindan doldurulmuştur. Ancak eksik bilgiler ve uç değerler nedeniyle bazı veriler, veri setinden atılmıştır. 218 katılımcıdan elde edilen veriler ile AFA, 200 katılımcıdan elde edilen veriler ile DFA yapılmıştır.

DEOGÖÖ'nin geçerlik ve güvenirlik çalışmaları kapsamında tüm AFA süreçleri, madde analizi ve güvenirlik çalışmaları SPSS 21 programı kullanılarak hesaplanmıştır. AFA 
öncesi ilk olarak Kaiser-Meyer-Olkin (KMO) ve Bartlett Küresellik Testi yapılmıştır. Ardından ölçek faktörlerinin sayısı ve döndürme yöntemi belirlenerek hesaplanmıştır. Maddelerin faktörlere dağılımı ve döndürülmüş faktör yüküne ilişkin sonuçlar elde edilmiştir. Daha sonra LISREL 8.7 programı kullanılarak 200 veri ile DFA gerçekleştirilmiştir. Model sonuçları, iyi uyum ve kabul edilebilir uyum değerlerine göre karşılaştırılmıştır. Daha sonra toplam puanlar açısından üst \% 27 ile alt \% 27 arasında anlamlı bir fark olduğunu göstermek için madde analizi yapılmıştır. Son olarak, ölçeğin iç tutarlılığını ortaya koymak için Cronbach Alfa değeri ve faktörler arasındaki korelasyonlar hesaplanmıştır. DEOGÖÖ'nin geçerlik ve güvenirlik çalışmaları kapsamında yapılan analizler, bulgular bölümünde detaylı olarak verilmektedir.

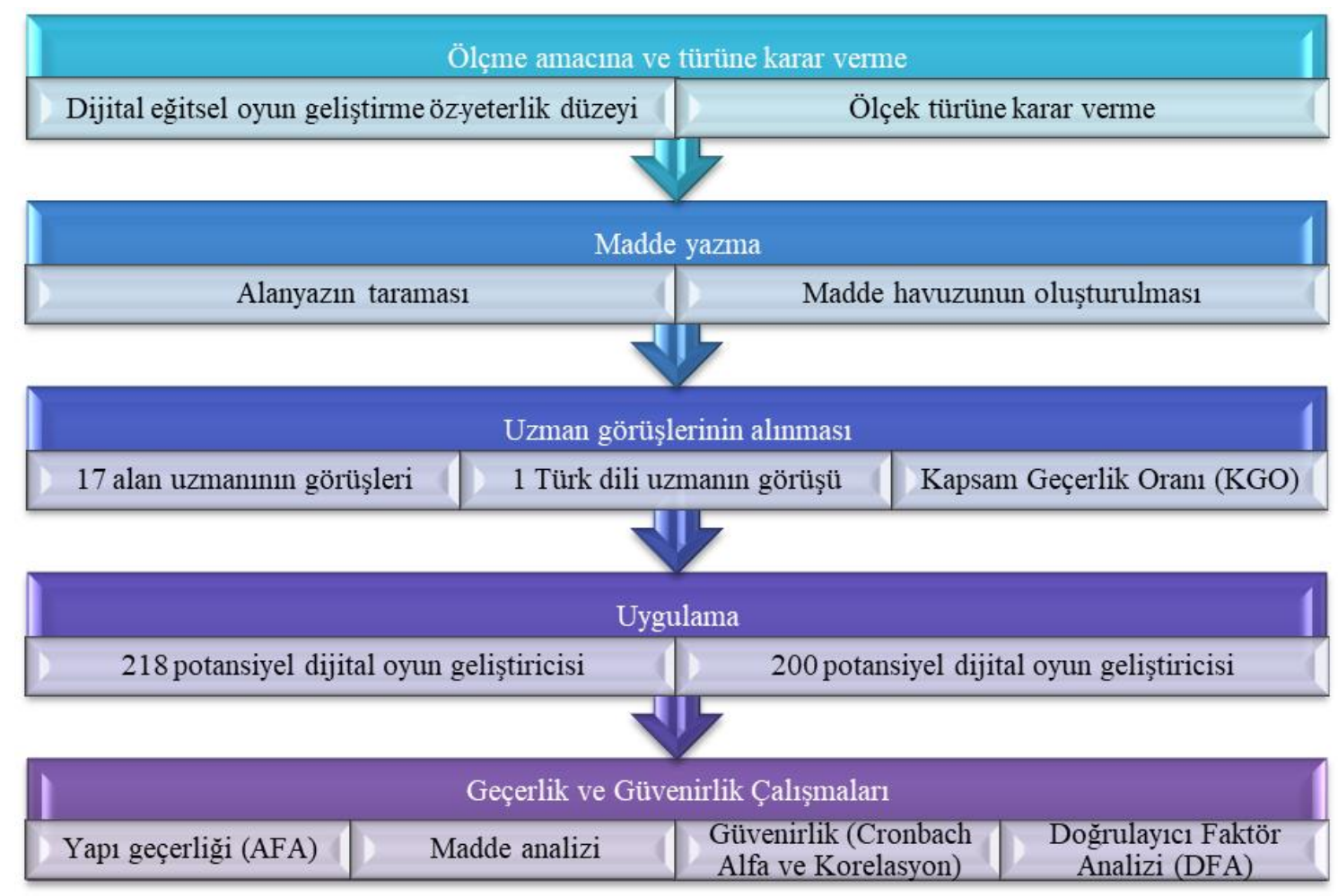

Şekil 1. DEOGÖÖ geliştirme sürecinin aşamaları

\section{Bulgular}

Bu bölümde Dijital Oyun Geliştirme Öz-Yeterlik Ölçeğinin geçerlik ve güvenilirlik çalışmaları sunulmuştur.

\section{Açımlayıcı Faktör Analizi}

AFA, 24 maddeyle yapılmıştır. Elde edilen verilerin AFA'ya uygunluğunu test etmek için KMO ve Bartlett Küresellik Testi yapılarak KMO değeri .965 (p = .000) olarak hesaplanmıştır. Can'a (2014) göre KMO değeri .70'den büyük ve anlamlı ise maddeler 
arasında ilişki olduğu ve örneklem büyüklüğünün analiz için iyi olduğu söylenebilir. Bu sonuca göre veriler AFA için uygundur.

Ölçeğin faktör sayısına karar vermek için AFA yapılmıştır. Analiz sonucunda öz değeri birden büyük olan iki faktör olduğu görülmüştür. Faktör analizinde bir alt boyutun öz değerinin en az \% 1 olması beklenmektedir (Aksu, Eser ve Güzeller, 2017; Seçer, 2015). Bu durum, Şekil 2'de sunulan yamaç grafiği incelenerek doğrulanmıştır.

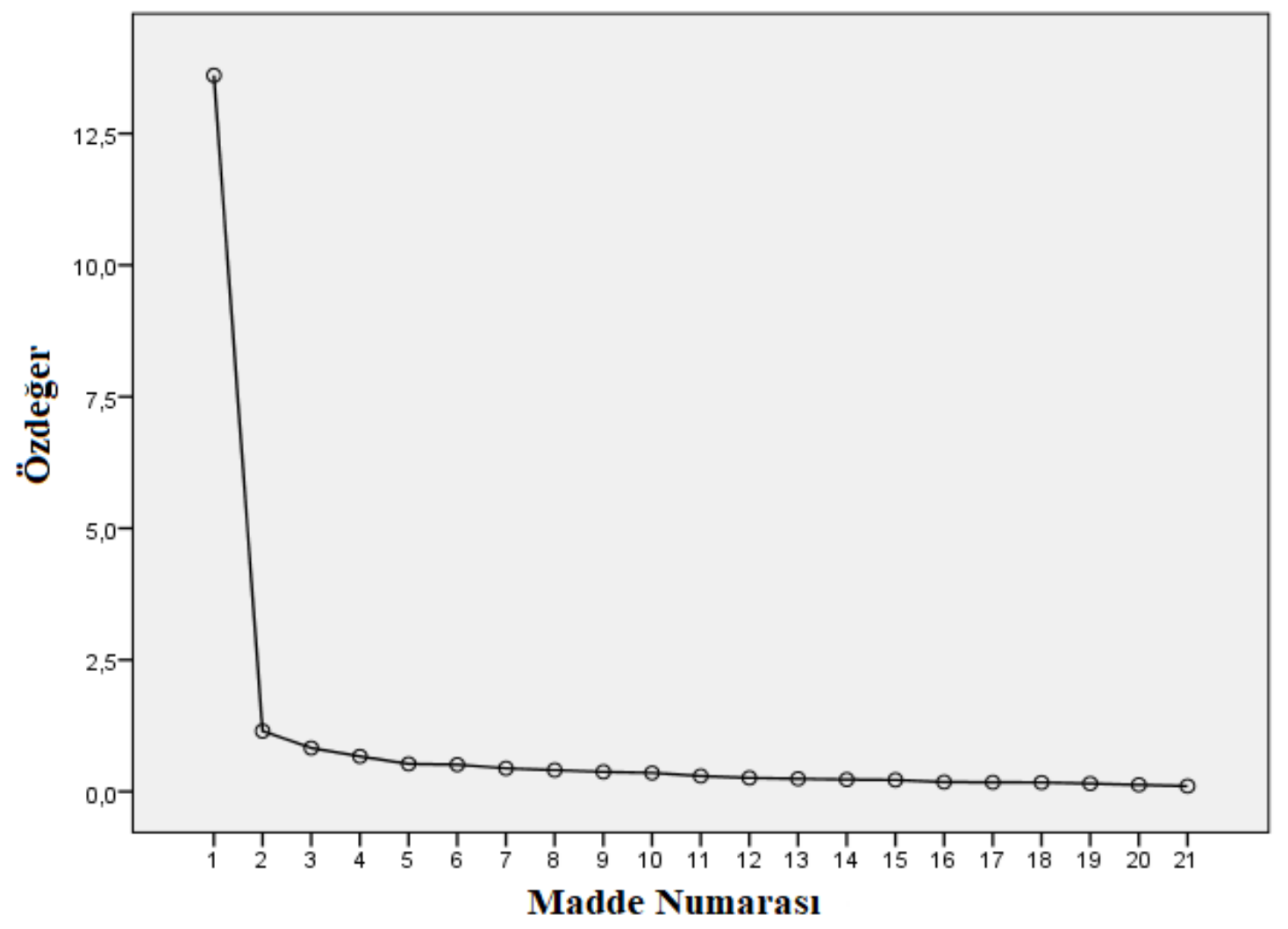

Şekil 2. DEOGÖÖ yamaç birikinti grafiği

Faktörlerin birbirinden bağımsız olması durumunda dik (varimax) döndürme yöntemi tercih edilmektedir (Ho, 2006). Dik döndürme yöntemlerinden biri olan varimax, faktörler arasındaki ilişkiyi ortadan kaldırması ve faktörleri daha net ortaya çıkarması nedeniyle tercih edilmektedir (Büyüköztürk, 2011; Can, 2014). Döndürme sonucunda madde yükleri .30'dan küçük ve bir maddenin birden fazla yüklenerek aralarında .1'den düşük yük değerinin olması (binişiklik) ise maddelerin ölçekten çıkarılmasını gerekmektedir (Çokluk, Şekercioğlu ve Büyüköztürk, 2012). Faktör yükü .30'dan daha düşük olan madde bulunmamaktadır. Ancak binişiklik nedeniyle 3 madde (6., 7. ve 13. maddeler) ölçekten çıkarılmıştır. $\mathrm{Bu}$ revizyonlara dayanarak AFA tekrarlanmıştır. Maddelerin faktörlere dağglımı ve döndürülmüş faktör yükleri Tablo 2'de verilmiştir. 


\begin{tabular}{|c|c|c|c|c|c|}
\hline \multirow[b]{2}{*}{$\begin{array}{l}\stackrel{0}{z} \\
\frac{0}{\pi} \\
\substack{\pi \\
\Sigma}\end{array}$} & \multirow[b]{2}{*}{ Maddeler } & \multirow[b]{2}{*}{ 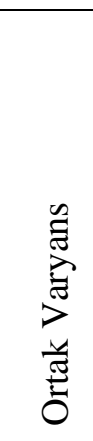 } & & \multicolumn{2}{|c|}{$\begin{array}{l}\text { Döndürülmüş } \\
\text { Faktör Yükleri }\end{array}$} \\
\hline & & & 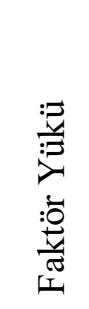 & 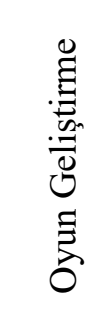 & 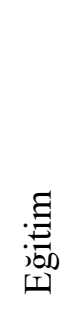 \\
\hline S15 & $\begin{array}{l}\text { Eğitsel oyun sahnelerini, görsel tasarım kurallarına uygun } \\
\text { olarak tasarlayabilirim. }\end{array}$ & .827 & .878 & .861 & \\
\hline S13 & $\begin{array}{l}\text { Mevcut bir dijital eğitsel oyun sahne tasarımını görsel tasarım } \\
\text { kurallarına göre düzeltebilirim. }\end{array}$ & .759 & .836 & .831 & \\
\hline S12 & $\begin{array}{l}\text { Eğitsel bir oyun tasarımı oluştururken, görsel tasarım } \\
\text { kurallarını kullanabilirim. }\end{array}$ & .748 & .839 & .815 & \\
\hline S17 & $\begin{array}{l}\text { Eğitsel bir oyun geliştirmek için kullanılabilecek araçları } \\
\text { sayabilirim. }\end{array}$ & .777 & .871 & .799 & \\
\hline S11 & $\begin{array}{l}\text { Bir dijital eğitsel oyunda bulunması gereken görsel tasarım } \\
\text { kurallarını sıralayabilirim. }\end{array}$ & .744 & .845 & .797 & \\
\hline S19 & $\begin{array}{l}\text { Bir dijital eğitsel oyun geliştirmek için uygun araçları } \\
\text { seçebilirim. }\end{array}$ & .771 & .868 & .794 & \\
\hline S16 & $\begin{array}{l}\text { Dijital eğitsel oyun sahne tasarımlarını görsel tasarım } \\
\text { kurallarına göre değerlendirebilirim. }\end{array}$ & .725 & .833 & .789 & \\
\hline S14 & Eğitsel oyun sahneleri için öykü yaprakları hazırlayabilirim. & .693 & .812 & .776 & \\
\hline S18 & $\begin{array}{l}\text { Araçların teknik yeterliliklerini göz önünde bulundurarak, } \\
\text { geliştireceğim eğitsel oyunun hangi özelliklere sahip olacağını } \\
\text { tahmin edebilirim. }\end{array}$ & .762 & .870 & .762 & \\
\hline S21 & $\begin{array}{l}\text { Sahne tasarımları oluşturulmuş bir senaryoyu dijital eğitsel } \\
\text { oyuna dönüştürebilirim. }\end{array}$ & .672 & .816 & .721 & \\
\hline S8 & $\begin{array}{l}\text { Bir dijital eğitsel oyun senaryosu yazarken, oyun öğelerini } \\
\text { uygun bir şekilde kullanabilirim. }\end{array}$ & .729 & .854 & .699 & \\
\hline S20 & $\begin{array}{l}\text { Dijital eğitsel oyun geliştirmek için en az bir araç } \\
\text { kullanabilirim. }\end{array}$ & .659 & .812 & .678 & \\
\hline S9 & Eksik verilen bir dijital eğitsel oyun senaryosunu & .667 & .816 & .667 & \\
\hline
\end{tabular}



tamamlayabilirim.

Oluşturulan kazanıma ilişkin dijital eğitsel oyun senaryosu yazabilirim.

Bir dijital eğitsel oyun senaryosunun yazım aşamalarını siralayabilirim.

Bir dijital eğitsel oyun geliştirirken dijital eğitsel oyun tasarım modellerinden birini kullanabilirim.

Geliştirilen dijital eğitsel oyunun kullanışl1lığını uygun tekniklere göre test edebilirim.

Bir dijital eğitsel oyun tasarlamak için hedef kitlenin ihtiyaçları doğrultusunda kazanım oluşturabilirim.

Bir dijital eğitsel oyun tasarlamak için hedef kitlenin ihtiyaçlarını belirleyebilirim.

Dijital eğitsel oyun öğelerini oluşturulan kazanıma uygun şekilde kullanabilirim.

S4 Bir dijital eğitsel oyunu pedagojik açıdan inceleyebilirim.

.461

.324

.602

\section{Öz değer}

Ölçekten 3 madde çıkarıldıktan sonra Tablo 2'ye göre toplam varyansın \% 70.25'ini açıklayan 21 madde elde edilmiştir. Birinci faktör, ölçeğin toplam varyansının \% 46.35 'ini, ikinci faktör ise \% 23.9'unu açılamaktadır. Sosyal bilimlerde \% 40 ile \% 60 arasındaki toplam varyans değerinin yeterli olduğu iddia edilmekte ve bir faktörün ilgili olabilmesi için açılanan toplam varyansın en az \% 5'inin bu faktörle ilişkili olması gerekmektedir (Çokluk, Şekercioğlu ve Büyüköztürk, 2010; Tavşanc1l, 2002). Döndürülmüş faktör yükleri .602 ile .861 arasında değişmektedir. Maddelerin faktör yük değerlerinin .45 ve üzerinde olması, maddenin ilgili faktörü iyi ölçtüğü anlamına gelmektedir (Büyüköztürk, 2011). Buradan yola çıkarak DEOGÖÖ'de yer alan maddelerin faktörlerle ilişkisinin iyi düzeyde olduğu söylenebilir.

Ölçek, Şekil 2'de verilen yamaç birikinti grafiğinde görüldüğü gibi iki faktörden oluşmaktadır. Ölçeğin birinci faktörü olan Oyun Geliştirme 17 maddeden, ikinci faktörü olan Eğitim ise 4 maddeden oluşmaktadır. 
Ölçme aracı ile ölçülecek değişkenin gruplara göre farklılık gösterip göstermemesi yapı geçerliliğinin kanıtı olarak kabul edilebilir (Baykul, 2000). Bu nedenle, katılımcıların DEOGÖ puanlarının DEOG eğitimine katılma durumlarına ve dijital eğitsel oyun geliştirme deneyimlerine göre farklılık gösterip göstermediği incelenmiştir. Ayrıca, farklılıkların etki büyüklüğünü görmek için Cohen's $d$ değeri hesaplanmıştır. Cohen's $d$ değeri -1 ile +1 arasında değişmekle birlikte 1'den büyük olabilir (Riazi, 2016). Ayrıca etki büyüklüğü 0.2 küçük etki, 0.5 orta etki ve 0.8 büyük etki olarak sınıflandırılmaktadır (Cohen, 1988). Tablo 3, katılımcıların dijital eğitsel oyun geliştirme eğitimine katılıp katılmama durumlarına göre DEOGÖ düzeyleri arasındaki farkları göstermektedir.

Tablo 3. Oyun geliştiricilerinin dijital eğitsel oyun geliştirme eğitimi alma durumlarına göre DEOGÖ düzeyleri

\begin{tabular}{|c|c|c|c|c|c|c|c|c|}
\hline DEOGÖÖ & DEOG & & & & & & & Etki \\
\hline \multirow[t]{2}{*}{ Boyutları } & Eğitimine & $\mathrm{N}$ & $\bar{X}$ & SS & $\mathrm{t}$ & sd & $\mathrm{p}$ & Büyüklüğü \\
\hline & Katılma & & & & & & & Cohen's $d$ \\
\hline \multirow[t]{2}{*}{ Eğitim } & Evet & 66 & 17.06 & 2.595 & 5.282 & 216 & .000 & 0.80 \\
\hline & Hayır & 152 & 14.71 & 3.171 & & & & \\
\hline \multirow{2}{*}{$\begin{array}{l}\text { Oyun } \\
\text { Geliştirme }\end{array}$} & Evet & 66 & 6633 & 1166 & 6003 & 216 & 000 & 092 \\
\hline & Hayır & 152 & 54.15 & 14.57 & & & & \\
\hline \multirow[t]{2}{*}{ Toplam } & Evet & 66 & 83.39 & 13.61 & 6.151 & 216 & .000 & 0.94 \\
\hline & Hayır & 152 & 68.86 & 16.95 & & & & \\
\hline
\end{tabular}

Katılımcıların dijital eğitsel oyun eğitimi alma durumuna göre DEOGÖ’nin değişip değişmediğini incelemek için bağımsız grup $\mathrm{t}$ testi yapılmıştır. Elde edilen sonuçlar neticesinde, oyun geliştiricilerin DEOGÖ puanlarına göre hem ölçeğin toplamı hem de ölçeğin alt boyutlarında eğitime katılanlar lehine anlamlı farklılık bulunmuştur $(\mathrm{p}<.05)$. Anlamlı farklılıklar, ölçeğin toplamı ve iki alt boyut için büyük etki büyüklüğüne sahiptir (d>0.8). Tablo 4, katılımcıların DEOGÖ düzeylerinin dijital eğitsel oyun geliştirme 
deneyimlerine göre farklılık gösterip göstermediğini belirlemek için bağımsız grup t-testi sonuçlarını vermektedir.

Tablo 4. Oyun geliştiricilerinin dijital eğitisel oyun geliştirme deneyimlerine göre DEOGÖ düzeyleri

\begin{tabular}{|c|c|c|c|c|c|c|c|c|}
\hline DEOGÖÖ & DEO & & & & & & & Etki \\
\hline \multirow[t]{2}{*}{ Boyutları } & Geliştirme & $\mathrm{N}$ & $\bar{X}$ & ss & $\mathrm{t}$ & sd & $\mathrm{p}$ & Büyüklüğü \\
\hline & & & & & & & & Cohen's $d$ \\
\hline \multirow[t]{2}{*}{ Eğitim } & Evet & 78 & 17.01 & 2.371 & 6.428 & 216 & .000 & 0.86 \\
\hline & Hayır & 140 & 14.54 & 3.252 & & & & \\
\hline \multirow[t]{2}{*}{ Oyun Geliştirme } & Evet & 78 & 66.30 & 10.572 & 7.616 & 216 & .000 & 1.02 \\
\hline & Hayır & 140 & 53.12 & 14.799 & & & & \\
\hline \multirow[t]{2}{*}{ Toplam } & Evet & 78 & 83.32 & 12.277 & 7.777 & 216 & .000 & 1.43 \\
\hline & Hayır & 140 & 67.66 & 17.229 & & & & \\
\hline
\end{tabular}

DEOGÖ düzeyi, katılımcıların oyun geliştirme deneyimlerine göre bağımsız grup t testi ile değerlendirilmiştir. Sonuçlara göre oyun geliştirme deneyimi olanların, deneyimi olmayanlara kıyasla DEOGÖ düzeylerinin anlamlı derecede yüksek olduğu görülmüştür $(\mathrm{p}<.05)$. Ölçeğin toplamı ve iki alt boyut için anlamlı farklılıklar büyük bir etkiye sahiptir $(d>0.8)$.

\section{Madde Analizi}

DEOGÖÖ’ne madde analizi yapabilmek için ölçekten alınan ham puanlar küçükten büyüğe sıralanmıştır. Daha sonra, en düşük ve en yüksek puanlara sahip 118 katılımcı ile alt \% 27 ve üst \% 27'lik gruplar oluşturulmuştur. Analiz sonuçlarına göre DEOGÖÖ'den elde edilen toplam puanlar açısından üst \% 27 ile alt \% 27 grupları arasında istatistiksel olarak anlamlı

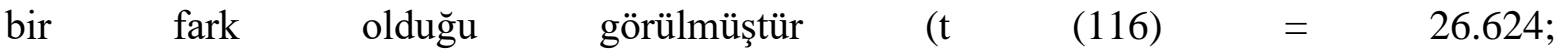
$\mathrm{p}=.000)$. Ancak ölçeğin her bir maddesi için üst ve alt grup puan ortalamaları arasındaki farkın $\mathrm{t}$ değeri hesaplanarak Tablo 5 'te sunulmuştur. Ayrıca madde-toplam korelasyonları .567 ile .862 arasındadır. Bu değerler bazı maddelerin orta $(.30-.70)$, bazılarının ise yüksek 
Ö. Kelleci ve T. Kulaksız / Pamukkale Üniversitesi Eğitim Fakültesi Dergisi, 52, 1-30, 2021

(.70 - 1.00) madde testi korelasyonlarına sahip olduğunu göstermektedir (Büyüköztürk, 2011).

Tablo 5. Ölçeğin alt \% 27 ve üst \% 27’lik grupları için bağımsız t-testi sonuçları

\begin{tabular}{|c|c|c|c|c|c|c|c|}
\hline $\begin{array}{l}\text { Madde } \\
\text { No }\end{array}$ & Grup & $\mathrm{N}$ & $\bar{X}$ & ss & $\mathrm{sd}$ & $\mathrm{t}$ & $\mathrm{p}$ \\
\hline \multirow{2}{*}{ s1 } & Üst grup & 59 & 4.81 & .472 & \multirow{2}{*}{116} & \multirow{2}{*}{13.355} & \multirow{2}{*}{.000} \\
\hline & Alt grup & 59 & 3.11 & .852 & & & \\
\hline \multirow{2}{*}{$\mathrm{s} 2$} & Üst grup & 59 & 4.69 & .701 & \multirow{2}{*}{116} & 10.670 & \multirow{2}{*}{.000} \\
\hline & Alt grup & 59 & 3.11 & .892 & & & \\
\hline \multirow{2}{*}{ s3 } & Üst grup & 59 & 4.66 & .575 & \multirow{2}{*}{116} & 11.318 & \multirow{2}{*}{.000} \\
\hline & Alt grup & 59 & 3.01 & .955 & & & \\
\hline \multirow{2}{*}{ s4 } & Üst grup & 59 & 4.40 & .790 & \multirow{2}{*}{116} & \multirow{2}{*}{9.018} & \multirow{2}{*}{.000} \\
\hline & Alt grup & 59 & 2.83 & 1.082 & & & \\
\hline \multirow{2}{*}{ s5 } & Üst grup & 59 & 4.54 & .702 & \multirow{2}{*}{116} & \multirow{2}{*}{14.055} & \multirow{2}{*}{.000} \\
\hline & Alt grup & 59 & 2.54 & .837 & & & \\
\hline \multirow{2}{*}{ s6 } & Üst grup & 59 & 4.66 & .512 & \multirow{2}{*}{116} & 14.108 & \multirow{2}{*}{.000} \\
\hline & Alt grup & 59 & 2.71 & .929 & & & \\
\hline \multirow{2}{*}{ s7 } & Üst grup & 59 & 4.71 & .493 & \multirow{2}{*}{116} & 15.431 & \multirow{2}{*}{.000} \\
\hline & Alt grup & 59 & 2.49 & .989 & & & \\
\hline \multirow{2}{*}{ s8 } & Üst grup & 59 & 4.77 & .418 & \multirow{2}{*}{116} & 17.369 & \multirow{2}{*}{.000} \\
\hline & Alt grup & 59 & 2.54 & .896 & & & \\
\hline s9 & Üst grup & 59 & 4.72 & .448 & 116 & 16.815 & .000 \\
\hline
\end{tabular}




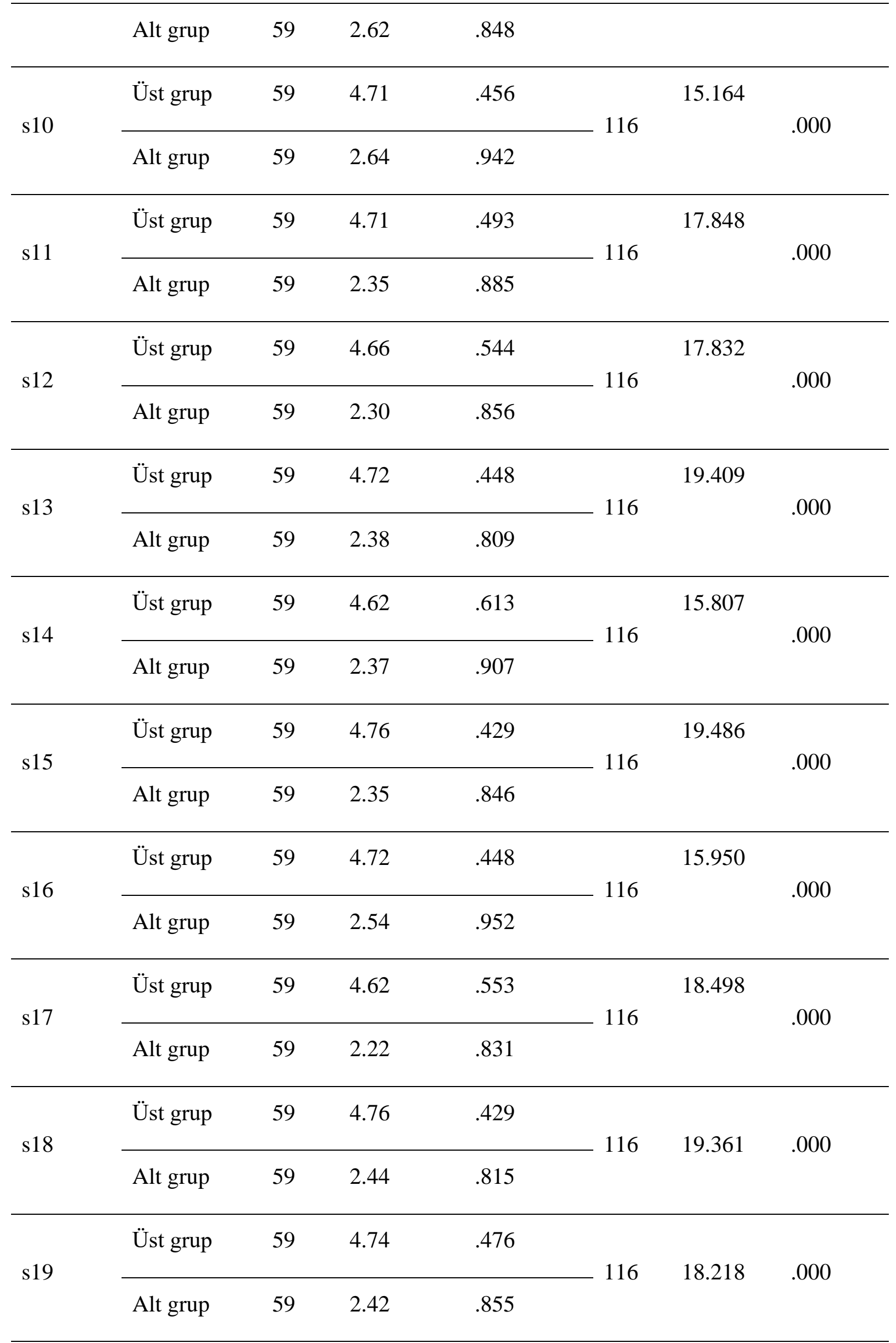




\begin{tabular}{|c|c|c|c|c|c|c|c|}
\hline \multirow{2}{*}{ s20 } & Üst grup & 59 & 4,77 & .617 & \multirow{2}{*}{116} & \multirow[t]{2}{*}{16.756} & \multirow{2}{*}{.000} \\
\hline & Alt grup & 59 & 2,44 & .876 & & & \\
\hline \multirow{2}{*}{ s21 } & Üst grup & 59 & 4,64 & .580 & \multirow{2}{*}{116} & \multirow{2}{*}{15.021} & \multirow{2}{*}{.000} \\
\hline & Alt Grup & 59 & 2,49 & .935 & & & \\
\hline
\end{tabular}

\section{Güvenirlik}

DEOGÖÖ'nin güvenilirlik çalışması kapsamında iç tutarlılık katsayıları hesaplanmıştır. İlk faktör olan Eğitim için Cronbach Alfa katsayısı .836, Oyun Geliştirme olan ikinci faktör için .973 ve ölçek toplamı için .972 'dir. .70 ve üstü iç tutarlık katsayıları güvenilir kabul edildiğinden (Büyüköztürk, 2011; Fraenkel, Wallen ve Hyun, 2012) hesaplanan güvenirlik katsayılarının yeterli olduğu söylenebilir. Ayrıca Oyun Geliştirme ile Eğitim faktörü arasında anlamlı bir ilişki vardır $(\mathrm{r}=.739, \mathrm{p}=<.00 ; \mathrm{N}=218)$. Bu bakımdan boyutlar arasında kabul edilebilir bir güvenirlik görülmektedir.

\section{Doğrulayıcı Faktör Analizi}

AFA ile ilişkili faktör yapısının geçerliliğini test etmek için DFA yapılmıştır. Modelin uygunluğunu belirlemek için Schermelleh-Engel, Moosbrugger ve Müller (2003) tarafindan önerilen $\chi 2$ / df, RMSEA, SRMR, NFI, CFI, GFI ve AGFI değerler referans alınmıştır. İyi uyum ve kabul edilebilir uyum değerlerine göre modelin sonuçları Tablo 6'da verilmiştir. 


\begin{tabular}{lccc}
\hline Indeks & İyi uyum & $\begin{array}{c}\text { Kabul edilebilir } \\
\text { uyum }\end{array}$ & Model uyumu değerleri \\
\hline$\chi 2 / \mathrm{df}$ & $0 \leq \chi 2 / \mathrm{sd} \leq 2$ & $2<\chi 2 / \mathrm{sd} \leq 3$ & 2.047 \\
RMSEA & $0 \leq \mathrm{RMSEA} \leq .05$ & $05<\mathrm{RMSEA} \leq .08$ & 0.073 \\
SRMR & $0 \leq \mathrm{SRMR} \leq .05$ & $.05<\mathrm{SRMR} \leq .10$ & 0.039 \\
NFI & $.95 \leq \mathrm{NFI} \leq 1.00$ & $.90 \leq \mathrm{NFI}<.95$ & 0.97 \\
NNFI & $.97 \leq \mathrm{NNFI} \leq 1.00$ & $.95 \leq \mathrm{NNFI}<.97$ & 0.98 \\
CFI & $.97 \leq \mathrm{CFI} \leq 1.00$ & $95 \leq \mathrm{CFI}<.97$ & 0.98 \\
GFI & $.95 \leq \mathrm{GFI} \leq 1.00$ & $.90 \leq \mathrm{GFI}<.95$ & 0.84 \\
AGFI & $.90 \leq \mathrm{AGFI} \leq 1.00$ & $.85 \leq \mathrm{AGFI}<.90$ & 0.80 \\
\hline
\end{tabular}

Tablo 6'da görülen modelin kabul edilebilir ve mükemmel uyum indeksi değerleri ile DFA'dan elde edilen uyum indeksi değerleri ile karşılaştırıldığında $\chi^{2} / \mathrm{sd}=2.047(\mathrm{p}=<.01)$ olarak belirlenmiştir. RMSEA $=.073 ;$ SRMR $=.039$; $\mathrm{NFI}=.97, \mathrm{NNFI}=.98, \mathrm{CFI}=.98 ; \mathrm{GFI}=.84$ ve $\mathrm{AGFI}=.80$ 'dir. Elde edilen sonuçlar mükemmel uyum değerlerine sahip olmasalar bile kabul edilebilirdir. Bu bulgular, Şekil 3 'te görüldüğü gibi DEOGÖÖ'nin faktör yapısını doğrulamaktadır. 


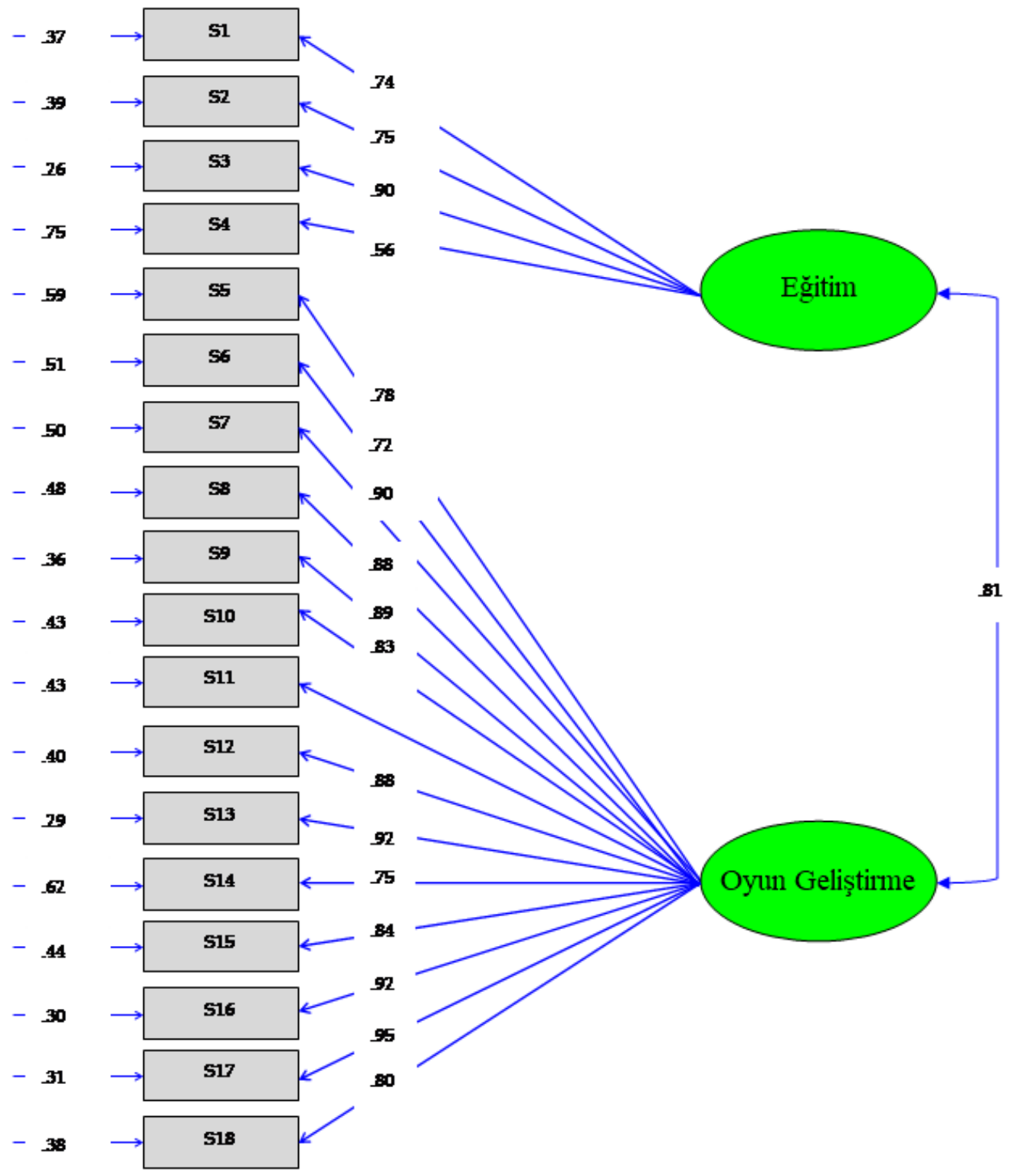

$$
\chi^{2}=376.77, \mathrm{sd}=184, p=0.00000, \mathrm{RMSEA}=0.073
$$

Şekil 3. Doğrulayıcı Faktör Analizi Sonuçları

\section{Sonuç ve Tartışma}

Bu çalışmada, geçerli ve güvenilir Dijital Eğitsel Oyun Geliştirme Öz-Yeterlik Ölçeğinin geliştirilmesi amaçlanmıştır. Ayrıca oyun geliştiricilerin DEO geliştirme eğitimi alma durumları ve DEO geliştirme deneyimlerine göre DEO geliştirme öz-yeterlik düzeyleri belirlenmiştir.

$\mathrm{Bu}$ çalışmadan elde edilen geçerlik ve güvenirlik analizi sonuçları, DEOGÖÖ'nin potansiyel DEO geliştiricilerin DEOGÖdüzeylerini ölçmek için kullanılabileceğini ortaya koymaktadır. Ayrıca DEOGÖÖ'den elde edilen sonuçlara göre DEO geliştirme eğitimine 
katılımın ve DEÖ geliştirme deneyiminin oyun geliştiricilerin DEO geliştirme öz-yeterlik düzeylerini artırdığı ortaya çıkmıştır. Bandura (1997) öz-yeterliği etkileyen temel faktörlerden birinin doğrudan deneyimler olduğunu belirtmiştir. Ayrıca bireylerin pozitif deneyimleri ve eğitim almaları öz-yeterliklerini olumlu yönde etkileyebilmektedir (Tschannen-Moran ve Hoy, 2007). DEOGÖÖ'den elde edilen bulgular bu çalışmaları desteklemektedir.

DEOGÖÖ 21 maddeden oluşmaktadır. DEOGÖÖ'nün maddeleri 5'li Likert türündedir. Her maddeye katılım durumu "Tamamen katılıyorum" (5), "Katılıyorum" (4), "Kararsızım" (3), "Katılmıyorum" (2) ve "Tamamen katılmıyorum" (1) olarak derecelendirilmektedir. DEOGÖÖ iki faktörden oluşmaktadır. Eğitim olarak tanımlanan birinci faktör 4 maddeden oluşmaktadır. Oyun geliştirme olarak tanımlanan ikinci faktör ise 17 maddeden oluşmaktadır. Ölçekten en az 21 en fazla 105 puan alınabilmektedir. Ölçekten alınan puanlar arttıkça bireylerin DEO geliştirme öz-yeterlik düzeyleri yükselmektedir. DEOGÖÖ ekte verilmiştir.

DEOların etkili bir şekilde geliştirilmesi için ilgili çalışmalarda analiz, tasarım, geliştirme, uygulama ve değerlendirme aşamalarını içeren dijital eğitsel oyun geliştirme modelleri önerilmektedir (Akgün ve diğerleri, 2011; Akıllı ve Çăğltay, 2006; Zin ve diğerleri, 2009). Bu aşamalara ek olarak Kocadere ve diğerleri (2019) çalışmalarında önerdikleri dijital eğitsel oyun geliştirme modeline analiz aşamasından önce ön analiz aşamasını da dahil etmiştir. Bu modellerin ön analiz/analiz safhalarında gerçekleşmesi istenen görevlerin DEOGÖÖ’nin eğitim boyutundaki madde içerikleri ile büyük ölçüde örtüştüğü söylenebilir. Yine DEOGÖÖ’nin oyun geliştirme boyutundaki madde içeriklerinin de dijital eğitsel oyun geliştirme modellerindeki tasarım, geliştirme ve uygulama safhalarında gerçeklemesi istenen görevler ile benzeştiği söylenebilir. Değerlendirme safhasında gerçekleşen görevler ise, DEOGÖÖ’nin hem eğitim hem de oyun geliştirme boyutlarında yer almaktadır. Nitekim dijital eğitsel oyun geliştirme modellerinde de değerlendirme safhası dijital eğitsel oyun geliştirme sürecinin bütününde yer almaktadır. Buna göre DEOGÖÖ’nin genel olarak bir dijital eğitsel oyun geliştirirken kullanılacak modeller ile uyumlu olduğu söylenebilir. Yine ilgili çalışmalar belli bir konuya ilişkin özyeterlik düzeyi yüksek bireylerin, genellikle o konuya yönelik daha iyi performans sergilemeye eğilimli olduğunu ortaya koymaktadır (Usher, Li, Butz ve Rojas, 2019). Bu açıdan düşünüldüğünde DEOGÖÖ’den elde edilen sonuçlara göre DEO geliştirme özyeterlik düzeyi yüksek dijital eğitsel oyun geliştiricilerin, daha etkili dijital eğitsel oyunlar 
geliştirebileceği de ifade edilebilir. Aynı zamanda ilgili çalışmalarda dijital eğitsel oyun geliştirme eğitimi kapsamında kazanılması öngörülen beceriler de (Kelleci ve Kulaksız, 2017; 2018a; 2018b) DEOGÖÖ’nin maddelerinde belirtilen yeterliklerle parallelik göstermektedir.

Dijital eğitsel oyunların kalitesinin arttırılması için nitelikli DEO geliştiricilerin sayısı artırılmalıdır. DEO geliştirme eğitimleri/kursları, potansiyel DEO geliştiricilerin eğitilmesini sağlayarak, DEOGÖÖ’nin yaygın etkisini artırabilir. Bu nedenle ileride yapılacak çalışmalarda DEOGÖÖ’nin farklı özellikteki katılımcı gruplarına uygulanması önerilmektedir. DEOGÖÖ ile gerçekleşen araştırma sürecine farklı değişkenler dahil edilerek DEOGÖ’yi etkileyen faktörlerin araştırılması ve DEO geliştirme eğitimlerinin/kurslarının etkililiğini test etmek için DEOGÖÖ’nin kullanılması önerilmektedir.

Etik Kurul İzin Bilgisi: Bu araştırma, Hasan Kalyoncu Üniversitesi, Sosyal Bilimler Enstitüsü etik kurulunun 16/03/2020 tarihli 2003160012 sayll kararl ile alınan izinle yürütülmüştür.

Çılkar Çatışması: Yazarların beyan edeceği bir çıkar çatışması yoktur.

Yazar Katkısı: Birinci yazar, problem durumunun belirlenmesi, literatür taraması, verilerin toplanması ve analizi aşamalarında; ikinci yazar, problem durumunun belirlenmesi, araştırma yönteminin tasarımı, verilerin toplanması ve analizi aşamalarında çalışmaya katkı sağlamıştır. Ayrıca her iki yazar da tartışma ve raporlama aşamasında çalışmaya katkı vermişstir. 


\section{Kaynakça}

Akgün, E., Nuhoğlu, P., Tüzün, H., Kaya, G. ve Çınar, M. (2011). Bir eğitsel oyun tasarımı modelinin geliştirilmesi. Ĕgitim Teknolojisi Kuram ve Uygulama, 1(1), 41-61.

Akıllı, K. G., \& Çağıltay, K. (2006). An instructional design/development model for the creation of game-like learning environments the FIDGE model. In M. Pivec (Ed.), Affective and emotional aspects of human-computer interaction: Game-based and innovative learning approaches (pp. 93-112). Amsterdam, Netherlands: IOS Press.

Aksu, G., Eser, M. T. ve Güzeller, C. O. (2017). Açımlayıcı ve doğrulayıcı faktör analizi ile yapısal eşitlik uygulamaları. Ankara: Detay Yayıncılık.

Aleem, S., Capretz, L. F., \& Ahmed, F. (2016). Critical success factors to improve the game development process from a developer's perspective. Journal of Computer Science and Technology, 31(5), 925-948.

An, Y. J., \& Cao, L. (2017). The effects of game design experience on teachers' attitudes and perceptions regarding the use of digital games in the classroom. TechTrends, 61(2), 162-170.

An, Y-J., Haynes, L, D'Alba, A., \& Chumney, F. (2016). Using educational computer games in the classroom: Science teachers' experiences, attitudes, perceptions, concerns, and support needs. Contemporary Issues in Technology \& Teacher Education, 16(4), 415-433.

Aşkar, P. ve Dönmez, O. (2004). Eğitim yazılımı geliştirme öz-yeterlik algısı ölçeği. Eğitim Bilimleri ve Uygulama, 3(6), 259-274.

Bakaç, E. ve Özen, R. (2015). Materyal tasarımı öz-yeterlik inancı ölçeği: Geçerlik ve güvenirlik çalışması. International Journal of Human Sciences, 12(2), 461-476.

Bandura, A. (1997). Self-efficacy: The exercise of self-control. New York: Freeman. 
Baykul, Y. (2000). Ĕgitimde ve psikolojide ölçme (3. Basım). Ankara: Pegem Akademi.

Brom, C., Dechterenko, F., Sisler, V., Hlavka, Z., \& Lukavsky, J. (2018). Does motivation enhance knowledge acquisition in digital game-based and multimedia learning? A review of studies from one lab. In Göbel S. et al. (Eds.) Serious Games. JCSG 2018. Lecture Notes in Computer Science, vol 11243. Springer, Cham.

Brown, Q., Lee, F., \& Alejandre, S. (2009, April). Emphasizing soft skills and team development in an educational digital game design course. In Fourth International Conference on the Foundations of Digital Games, 240-247.

Bryman, A., \& Cramer, D. (1999). Quantitative data analysis with SPSS release 8 for Windows: A guide for social scientists. London: Routledge.

Büyüköztürk, Ş. (2011). Sosyal bilimler için veri analizi el kitabı (15. Basım) Ankara: Pegem Akademi.

Büyüköztürk, Ş., Çakmak, K. Ç., Akgün, E. Ö., Karadeniz, Ş. ve Demirel, F. (2015). Bilimsel araştırma yöntemleri (19. Basım). Ankara: Pegem Akademi.

Byun, J., \& Joung, E. (2018). Digital game-based learning for K-12 mathematics education: A meta-analysis. School Science and Mathematics, 118, 113-126.

Cai, L., Liu, F., \& Liang, Z. (2010, December). The research and application of education game design model in teaching Chinese as a Foreign Language. In 2010 IEEE International Conference on Progress in Informatics and Computing (Vol. 2, pp. 1241-1245). China: IEEE.

Can, A. (2014). SPSS ile bilimsel araştırma sürecinde nicel veri analizi. Ankara: Pegem Akademi. 
Cherian, J., \& Jacob, J. (2013). Impact of self efficacy on motivation and performance of employees. International Journal of Business and Management, 8(14), 80-88.

Chen, C-H., Shih, C.-C., \& Law, V. (2020). The effects of competition in Digital GameBased Learning (DGBL): A meta-analysis. Educational Technology Research and Development, 68(3), 1-19. doi: 10.1007/s11423-020-09794-1

Cohen, J. (1988). Statistical power analysis for the behavioral sciences (2nd Edition). Hillsdale, NJ: Lawrence Erlbaum.

Cronbach, L. J. (1990). Essentials of psychological testing (5th Edition). New York: HarperRow.

Çokluk, Ö., Şekercioğlu, G. ve Büyüköztürk, Ş. (2010). Sosyal bilimler için çok değişkenli istatistik. Ankara: Pegem Yayınc1lık.

Çokluk, Ö., Şekercioğlu, G. ve Büyüköztürk, Ş. (2012). Sosyal bilimler için çok değişkenli SPSS ve LISREL uygulamaları. Ankara: Pegem A Yayıncılık.

DeVellis, R. F. (2003). Scale development: Theory and application (6th Edition). Thousand Oaks, CA: SAGE.

Fraenkel, J. R., Wallen, N. E., \& Hyun, H. H. (2012). How to design and evaluate research in education (8th Edition). New York, NY: McGraw Hill Education.

Gaudelli, W., \& Talyor, A. (2011). Modding the global classroom? Serious video games and teacher reflection. Contemporary Issues in Technology and Teacher Education, 11(1), 70-91.

Gros, B. (2015). Integration of digital games in learning and e-learning environments: Connecting experiences and context. In Digital Games and Mathematics Learning (pp. 35-53). Springer: Dordrecht. 
Gürbüz, S. ve Şahin, F. (2018). Sosyal bilimlerde araştırma yöntemleri (5. Basım). Ankara: Seçkin Yayıncılık.

Hava, K., \& Cakir, H. (2017). A systematic review of literature on students as educational computer game designers. In J. Johnston (Ed.), Proceedings of EdMedia 2017 (pp. 407-419). Washington, DC: Association for the Advancement of Computing in Education.

Hewner, M., \& Guzdial, M. (2010, March). What game developers look for in a new graduate: Interviews and surveys at one game company. 41st ACM Technical Symposium on Computer Science Education, Milwaukee, Wisconsin, USA.

Ho, R. (2006). Handbook of univariate and multivariate data analysis and interpretation with SPSS. New York: Taylor and Francis.

International Society for Technology in Education. (2017). ISTE standards for educators. Retrieved from http://www.iste.org/standards/for-educators.

Kelleci, Ö. ve Kulaksız, T. (2017, Ekim). Eğitsel Oyun Tasarlama Ĕgitiminin etkililiğinin değerlendirilmesi. 5. International Instructional Technologies and Teacher Education Symposium, İzmir, Türkiye.

Kelleci, Ö. ve Kulaksız, T. (2018a, Mayıs). Bir dijital eğitsel oyun tasarlama eğitiminin performansa dayalı olarak karşılaştırmalı değerlendirilmesi: Öğretmen adayı perspektifi. 12. International Computer \& Instructional Technologies Symposium (ICITS 2018), İzmir, Türkiye.

Kelleci, Ö., \& Kulaksız, T. (2018b, April). Developing an educational digital game design course. 8. World Conference On Educational Technology Researches, Batum, Georgia. 
Kocadere, S. A., Özhan, Ş. Ç., Bayrak, F. ve Kibar, P. N. (2019). Herkül'ün hikayesi: Bir eğitsel oyun modeli önerisi. Eğitim Teknolojisi Kuram ve Uygulama, 9(1), 230-250. doi: 10.17943/etku.451990.

Law, E. L., \& Sun, X. (2012). Evaluating user experience of adaptive digital educational games with activity theory. International Journal of Human Computer Studies, 70(7), 478-497. doi: 10.1016/j.ijhcs.2012.01.007.

Li, Q., Lemieux, C., Vandermeiden, E., \& Nathoo, S. (2013). Are you ready to teach secondary mathematics in the 21 st century? A study of preservice teachers' digital game design experience. Journal of Research on Technology in Education, 45(4), 309-337. doi:10.1080/15391523.2013.10782608.

Martins, A. R., \& Oliveira, L. R. (2018, June). Students as Creators of Educational GamesLearning to Use Simple Frameworks and Tools to Empower Students as Educational Game Designers. In EdMedia+ Innovate Learning (pp. 1210-1215). Association for the Advancement of Computing in Education (AACE).

McGill, M. M. (2008, November). Critical skills for game developers: An analysis of skills sought by industry. 2008 Conference on Future Play: Research, Play, Share. Toronto, Canada.

McMahon, M. T. (2009). Using the DODDEL model to teach serious game design to novice designers. Proceedings of Ascilite 2009 (pp. 646-653). Auckland, New Zealand. The University of Auckland, Auckland University of Technology \& Australasian Society for Computers in Learning in Tertiary Education.

Milli Eğitim Bakanlığı. (2020). Öğretmenlik mesleği genel yeterlikleri. http://oygm.meb.gov.tr/www/ogretmenlik-meslegi-genel-yeterlikleri/_ icerik/39 adresinden erişildi. 
Ö. Kelleci ve T. Kulaksız / Pamukkale Üniversitesi Eğitim Fakültesi Dergisi, 52, 1-30, 2021

Noemi, P., \& Maximo, S. H. (2014). Educational games for learning. Universal Journal of Educational Research, 2(3), 230-238. doi: 10.13189/ujer.2014.020305.

Prensky, M. (2001). Digital game-based learning. New York: McGraw Hill.

Prensky, M. (2008). Students as designers and creators of educational computer games: Who else? British Journal of Educational Technology,39(6), 1004-1019. doi:10.1111/j.1467-8535.2008.00823_2.x.

Ramalingam, V., \& Wiedenbeck, S. (1998). Development and validation of scores on a computer programming self-efficacy scale and group analyses of novice programmer self-efficacy. Journal of Educational Computing Research, 19(4), 367-381. doi: 10.2190/c670-y3c8-1tj1-ct3p.

Riazi, A. M. (2016). The Routledge encyclopedia of research methods in applied linguistics. NY: Routledge.

Sancar Tokmak, H., \& Özgelen, S. (2013). The ECE pre-service teachers' perception on factors affecting the integration of educational computer games in two conditions: Selecting versus redesigning. Educational Sciences: Theory and Practice, 13(2) Spring, 1345-1356.

Schermelleh-Engel, K., Moosbrugger, H., \& Müller, H. (2003). Evaluating the fit of structural equation models: Tests of significance and descriptive goodness-of-fit measures. Methods of Psychological Research Online, 8(2), 23-74.

Seçer, İ. (2015). Psikolojik test geliştirme ve uyarlama süreci. Ankara: Anı Yayıncılık.

Talan, T., Doğan, Y., \& Batdı, V. (2020). Efficiency of digital and nondigital educational games: A comparative meta-analysis and a meta-thematic analysis. Journal of Research on Technology in Education, 52(4), 474-514. doi:10.1080/15391523.2020.1743798. 
Tavşancıl, E. (2002). Tutumların ölçülmesi ve spss ile veri analizi. Ankara: Nobel Yayıncilik.

Tschannen-Moran, M., \& Hoy, A. W. (2007). The differential antecedents of self-efficacy beliefs of novice and experienced teachers. Teaching and Teacher Education, 23(6), 944-956. doi:10.1016/j.tate.2006.05.003.

Tüzün, H., Barab, S. A., \& Thomas, M. K. (2019). Reconsidering the motivation of learners in educational computer game contexts. Turkish Journal of Education, 8(2), 129159. doi: 10.19128/turje.546283.

Usher, E. L., Li, C. R., Butz, A. R., \& Rojas, J. P. (2019). Perseverant grit and self-efficacy: Are both essential for children's academic success? Journal of Educational Psychology, 111(5), 877-902. doi:10.1037/edu0000324.

Veneziano L., \& Hooper J. (1997). A method for quantifying content validity of healthrelated questionnaires. American Journal of Health Behavior, 21(1), 67-70.

Worthington, R. W., \& Whittaker, T. A. (2006). Scale development research: A content analysis and recommendations for best practices. The Counseling Psychologist, 34(6), 806-838. doi:10.1177/0011000006288127.

Yang, Y. T. C. (2015). Virtual CEOs: A blended approach to digital gaming for enhancing higher order thinking and academic achievement among vocational high school students. Computers $\quad \& \quad$ Education, $\quad 81, \quad 281-295$. doi:10.1016/j.compedu.2014.10.004.

Yien, J. M., Hung, C. M., Hwang, G. J., \& Lin, Y. C. (2011). A game-based learning approach to improving students' learning achievements in a nutrition course. Turkish Online Journal of Educational Technology, 10(2), 1-10. 
Ö. Kelleci ve T. Kulaksız / Pamukkale Üniversitesi Eğitim Fakültesi Dergisi, 52, 1-30, 2021

Zin, N. A., Jaafar, A., \& Yue, W. S. (2009). Digital Game-Based Learning (DGBL) model and development methodology for teaching history. WSEAS Transactions on Computers, 8(2), 322-333. 
Değerli katılımc1,

$\mathrm{Bu}$ ölçek, öğretmen adaylarının dijital eğitsel oyun geliştirme öz-yeterlik düzeylerini ölçmek amacıyla hazırlanmıştır. Araç 'Demografik Bilgiler' ve 'Dijital eğitsel oyun Geliştirmeye Yönelik Maddeler' olmak üzere iki bölümden oluşmaktadır. Maddeler, 'Tamamen katılmıyorum-Katılmıyorum- Kararsızım- Katılıyorum- Tamamen katıl1yorum' şeklindeki 5'li Likert tipi cevap formatına göre yanıtlanacaktır. 1: Tamamen katılmıyorum, 2:Katılmıyorum, 3: Kararsızım, 4:Katılıyorum, 5: Tamamen katılıyorum Ölçekteki maddelere cevap verirken size en uygun olan durumu düşünerek cevap vermeniz, değerlendirmemizin sağlıklı olabilmesi için önem teşkil etmektedir.

\begin{tabular}{|c|c|c|c|}
\hline & 12 & 3 & 45 \\
\hline $\begin{array}{l}\text { 1-Bir dijital eğitsel oyun tasarlamak için hedef kitlenin ihtiyaçlarını } \\
\text { belirleyebilirim. }\end{array}$ & & & \\
\hline $\begin{array}{l}\text { 2-Bir dijital eğitsel oyun tasarlamak için hedef kitlenin ihtiyaçları } \\
\text { doğrultusunda kazanım oluşturabilirim. }\end{array}$ & & & \\
\hline $\begin{array}{l}\text { 3-Dijital eğitsel oyun öğelerini oluşturulan kazanıma uygun şekilde } \\
\text { kullanabilirim. }\end{array}$ & & & \\
\hline 4-Bir dijital eğitsel oyunu pedagojik açıdan inceleyebilirim. & & & \\
\hline $\begin{array}{l}\text { 5-Bir dijital eğitsel oyun geliştirirken dijital eğitsel oyun tasarım } \\
\text { modellerinden birini kullanabilirim. }\end{array}$ & & & \\
\hline 6-Geliştirilen dijital eğitsel oyunun kullanışl1lığını uygun tekniklere göre test & & & \\
\hline
\end{tabular}




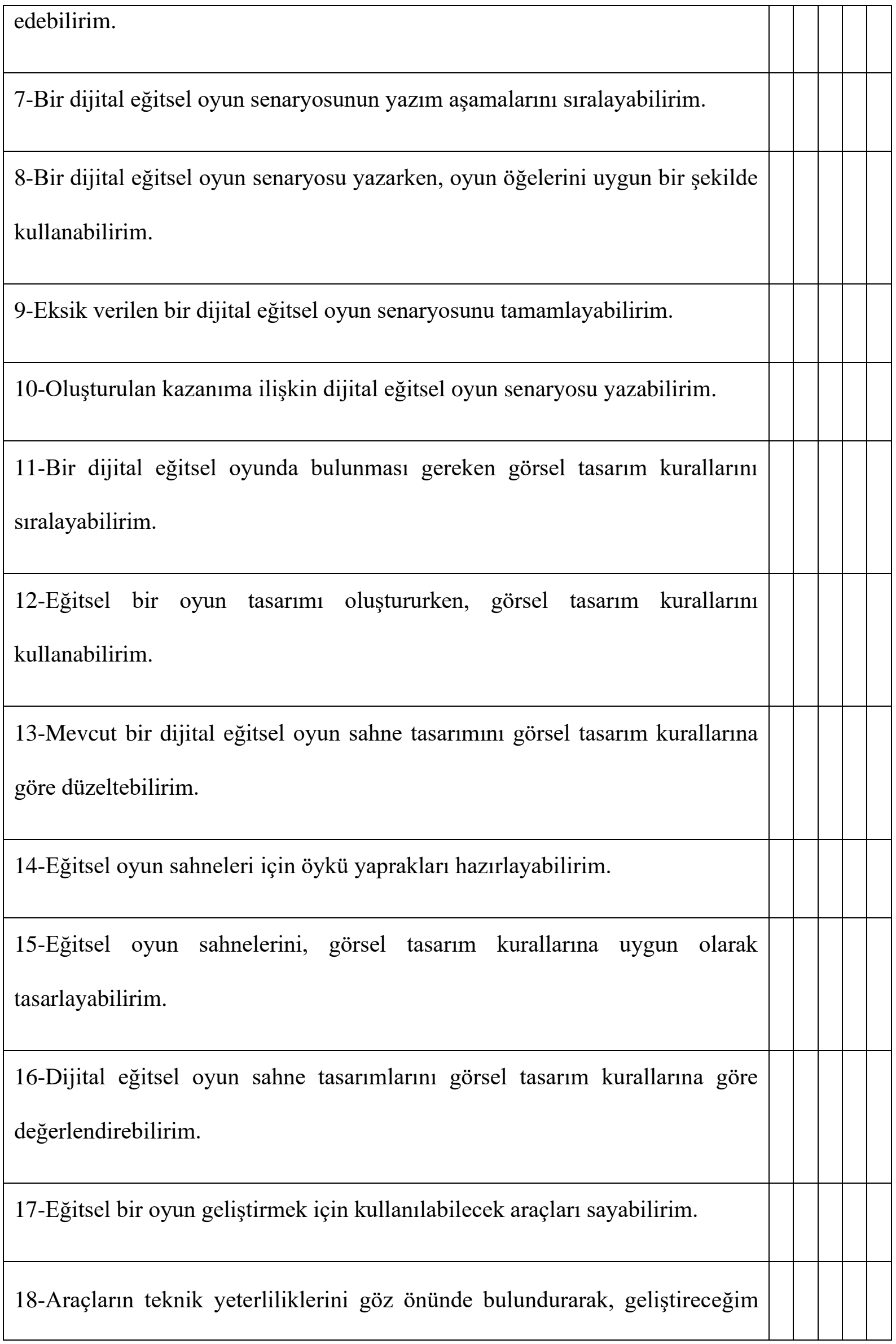


eğitsel oyunun hangi özelliklere sahip olacağını tahmin edebilirim.

19-Bir dijital eğitsel oyun geliştirmek için uygun araçları seçebilirim.

20-Dijital eğitsel oyun geliştirmek için en az bir araç kullanabilirim.

21-Sahne tasarımları oluşturulmuş bir senaryoyu dijital eğitsel oyuna dönüştürebilirim. 


\title{
Developing A Digital Educational Game Development Self-Efficacy Scale \\ Özge KELLECI ${ }^{*}$ Taibe KULAKSIZ ${ }^{* *}$
}

- Received: 08.04.2020 • Accepted: 01.09.2020 • Online First: 06.11.2020

\begin{abstract}
In this research, it is aimed to develop valid and reliable Digital Educational Game Development Self-Efficacy Scale (DEGDSS). In this context, the item pool was formed in line with the related literature. After that, the content validity ratio calculated for each item according to experts' opinions. The first draft of DEGDSS composed of 24 items was applied to 418 participants for validity and reliability studies. The data were analyzed through SPSS. Exploratory Factor Analysis (EFA) and Confirmatory Factor Analysis (CFA) were conducted to identify the construct validity of DEGDSS. The Kaiser-Meyer-Olkin (KMO) coefficient of the EFA was calculated as .965 and the Bartlett Sphericity Test was found significant $(\mathrm{p}=.000)$. As a result of the analysis of EFA, it was found that the 21-item-scale has a two-factor structure defined as Education and Game Development and these factors account for $70.25 \%$ of the total variance. As a result of item analysis conducted to determine the level of discrimination of items of the scale, the difference between the items was found to be significant at .01 level. Cronbach alpha reliability coefficient of the scale was calculated as .972 . As a consequence of the CFA, the model was acceptable.
\end{abstract}

Keywords: digital educational game, scale development, self-efficacy, digital educational game development self-efficacy, higher education

\section{Cited:}

Kelleci, Ö., \& Kulaksız, T. (2021). Developing a digital educational game development selfefficacy scale. Pamukkale University Journal of Education, 52, 1-30. doi:10.9779/pauefd.716426.

\footnotetext{
* Dr. Öğr. Üyesi, Hasan Kalyoncu University, Faculty of Education, Department of Curriculum and Instruction, ozge.kelleci@hku.edu.tr, ORCID: 0000-0001-6495-8320

** Dr. Sağlık Bilimleri Üniversitesi, taibekulaksiz@gmail.com, ORCID: 0000-0002-7960-7440
} 


\section{Introduction}

Technological developments in the twenty-first century have led to many socioeconomic, physical and psychological changes in societies. This digital transformation is also seen in education. New methods and approaches have been used in education in order to serve the purpose of this new society. In addition, the use of learning materials enriched with learning technologies, and learning environments have become more important. Among the new methods and approaches used in education, one of the effective methods that can be used to increase the success and motivation in education is the digital game-based learning approach (Brom, Dechterenko, Sisler, Hlavka, \& Lukavsky, 2018; Byun \& Joung, 2018; Chen, Shih, \& Law, 2020; Tüzün, Barab, \& Thomas, 2019; Yang, 2015; Yien, Hung, Hwang, \& Lin, 2011). In this method, learning is carried out more effectively through Digital Educational Games (DEGs) that can be personalized and designed in accordance with learning needs ( $\mathrm{Li}$, Lemieux, Vandermeiden, \& Nathoo, 2013; Prensky, 2001; Talan, Doğan, \& Batd1, 2020).

The DEGs, which have been used to bring academic skills to individuals since 1970 (Noemi \& Maximo, 2014), have been effective in acquiring abstract and conceptual knowledge, and as well as psychomotor and metacognitive skills (Law \& Sun, 2012). DEGs focus on learning by doing, and the transfer of knowledge to real-life (An \& Cao, 2017). In order for DEGs to be effective in education, it is important to develop them according to the learning needs of students, DEGs development models and standards (Li et al., 2013). It is also important that the potential DEG developers, who are teachers, programmers/engineers or pre-service teachers/students, have DEGs development competencies. As a matter of fact, one of the general competencies of the teachers, in the 21 st-century educational skills is that they can develop digital learning tools and materials in an effective way for students' needs (International Society for Technology in Education [ISTE], 2017; Ministry of National Education [MoNE], 2020).

Studies clearly demonstrate the contribution of DEGs to education, however, the low ability of teachers to develop DEGs and integrate of DEGs into the curriculum may prevent the use of DEGs as an effective tool in education (An, Haynes, D'Alba, \& Chumney, 2016; Gaudelli \& Talyor 2011; Gros, 2015; Hava \& Cakir, 2017; Li et al., 2013; Prensky, 2008; Sancar Tokmak \& Özgelen, 2013). In a study by Sancar Tokmak 
and Özgelen (2013), it was stated that each digital educational game did not answer the needs of the students, and teachers had difficulty in choosing an appropriate digital educational game for the educational content. In the study where Martins and Oliveira (2018) taught teachers how to design digital games, it was emphasized that the teachers who had high digital game development skills had high levels of motivation and learning. In the same study, it was also stated that teachers have low qualifications for developing DEGs. In the study of Hava and Cakir (2017), it was revealed that digital educational game development improved students' 21 st-century skills, problem-solving, creative thinking and programming skills, and the biggest obstacle in digital game development training was teachers' lack of experience and competence to develop DEGs.

On the other hand, the ability of an individual affects their judgment on the ability to accomplish the work. According to the social learning theory, self-efficacy is defined as the capacity of an individual to regulate and perform the necessary activities in order to demonstrate a certain performance (Bandura, 1997). An individual's selfefficacy perception towards a certain area enables them to be more willing to participate in activities related to the field and to deal with the problems they face more easily (Cherian \& Jacob, 2013). An individual's self-efficacy may not be at the same level all the time. It should be kept in mind that self-efficacy levels of teachers differ depending on the situation, subject or work, and should be evaluated accordingly. On the basis of this, it can be concluded that teachers with high self-efficacy in developing DEGs will have the ability to design more effective digital educational games for the learning needs of students.

Therefore, it is important to give digital educational game development training/course to teachers or other potential DEG developers. In this context, DEG developers are trained for designing DEGs to develop pedagogy, technique and design skills within the framework of an instructional design model (Brown, Lee, \& Alejandre, 2009). When the instructional design models used in digital educational game development training or course are examined, it is seen that digital game development process generally covers the stages of analysis, design, development, implementation, and evaluation (Akgün, Nuhoğlu, Tüzün, Kaya, \& Çınar, 2011; Akıllı \& Çağıltay, 2006; 
Cai, Liu, \& Liang, 2010; Kelleci \& Kulaksız, 2018b; Kocadere, Özhan, Bayrak, Kibar, 2019; McMahon, 2009; Zin, Jaafar, \& Yue, 2009). There are already studies to determine competencies related to game development (Aleem, Capretz, \& Ahmed, 2016; Hewner \& Guzdial, 2010; McGill, 2008). In these studies, two criteria were taken into consideration, the game development process, and the competencies that the game developers should have, both of them were discussed. It is seen that these competencies are generally in technical (programming), design and management dimensions.In addition, in order to develop DEGs effectively, it is important to carry out studies to identify and increase the competencies of potential DEG developers. In the digital educational game development courses are aimed to increase digital educational game development skills and self-efficacy levels. Hence, it is important to determine DEGDS levels of the potential DEG developers. However, the digital game development selfefficacy scale was not found when the literature examined. In the literature, there is Educational Software Development Self-Efficacy Scale (Aşkar \& Dönmez, 2004), Teaching Material Design Self-Efficacy Scale (Bakaç \& Özen, 2015), Computer Programming Self-Efficacy Scale (Ramalingam \& Wiedenbeck, 1998). However, these scales do not specifically measure the self-efficacy level of digital educational game development. Hence, the main aim of the study is to develop a valid and reliable Digital Educational Game Development Self-Efficacy Scale (DEGDSS). Also, it is aimed at determining game developers' DEGD self-efficacy levels according to their attending DEGD course and their experience in DEGD.

\section{Method}

\section{Participants}

The DEGDS scale developed within the scope of this study is for potential digital educational game developers. Ethical approval for this study has been provided by Hasan Kalyoncu University, Social Sciences, and the Humanities Institute of Research Ethics Committee (No: 2003160012). Participation in the research is voluntary. Accordingly, participants were included based on the purposeful sampling method for the study. For the purpose of the research, purposeful sampling is preferred when participants with certain characteristics are selected (Büyüköztürk, Çakmak, Akgün, Karadeniz, \& Demirel, 2015). In this context, it was decided that the participants will be 
composed of higher education students and graduates from Computer Education and Instructional Technology (CEIT), elemantary education and computer engineering department.Therefore, participants are thoses who took digital educational game development courses/training, those who have the necessary prerequisite skills for digital educational game development, or those who are working on this field. Accordingly, data were collected from 418 participants determined according to the purposeful sampling method in the 2018-2019 academic year. EFA conducted with 218 participants whose ages ranged from 18 to 43 years. CFA conducted with 200 participants whose ages ranged from 18 to 41 years. The demographic information of the participants is presented in Table 1. In the scale development studies, it is sufficient that the number of samples is at least five times more than the number of items (Bryman \& Cramer, 1999; Tavşancıl, 2002). In this study, since the item number of draft scale items was 24 , it can be said that the number of participants is appropriate.

Table 1. Demographic information of the participants

\begin{tabular}{|c|c|c|c|c|c|}
\hline & & \multicolumn{2}{|c|}{ EFA } & \multicolumn{2}{|c|}{ DFA } \\
\hline & & $\mathrm{N}$ & $\%$ & $\mathrm{~N}$ & $\%$ \\
\hline \multirow{3}{*}{ Gender } & Female & 101 & 46.33 & 118 & 59 \\
\hline & & & & & \\
\hline & Male & 117 & 53.67 & 82 & 41 \\
\hline \multirow{5}{*}{$\begin{array}{l}\text { Educational } \\
\text { Level }\end{array}$} & 1. Grade & 23 & 10.55 & 5 & 2.5 \\
\hline & 2. Grade & 48 & 22.02 & 37 & 18.5 \\
\hline & 3. Grade & 29 & 13.30 & 104 & 52 \\
\hline & 4. Grade & 58 & 26.61 & 29 & 14.5 \\
\hline & Graduate & 60 & 27.52 & 25 & 12.5 \\
\hline Total & & 218 & 100 & 200 & 100 \\
\hline
\end{tabular}




\section{Development of Instrument}

The steps that should be followed in the development of a measurement tool, in general, are deciding what is wanted to be measured with the measurement tool, writing the items, taking experts' opinions, implementation, performing validity and reliability studies (Büyüköztürk et al., 2015; DeVellis, 2003; Worthington \& Whittaker, 2006). The steps of the DEGDSS development process are summarized in Figure 1. In this context, first of all, it was decided that the DEGDS were wanted to be measured during the development of DEGDSS, and the type of the instrument was determined.

Secondly, the item pool was generated based on the literature review, the relevant models and instruments employed to develop DEGs considered. As a result of the examinations, the 21-item pool was formed by the researchers. It was decided that the type of instrument was a five-degree Likert-type scale. The reason for this was that Likert-type scales were used to determine the degree of participation of the participants to the items created for the variables to be measured such as belief, attitude, thought and behavior (DeVellis, 2003; Gürbüz \& Şahin, 2018). The response to the items on the scale varies between "I Totally Disagree" (1) and "I Totally Agree" (5). 21 items fivedegree Likert-type draft form was created.

As a third step, experts' opinions were gathered. In this step, the draft form was presented to 17 field experts who have in minimum master's degree in CEIT to be evaluated in terms of content. Those experts were chosen considering that they have the knowledge and skills required to develop DEGs, and experience of scale development. In order to receive the opinions of the experts on the items in the draft form, a form with the triple Likert response format (Necessary, Unnecessary and Revision) was prepared. They were also asked to elicit opinions and suggestions from the experts on the matter. Then, the revised DEGDSS draft form was sent 1 Turkish language expert to be evaluated in terms of language-expression. After that, Content Validity Ratio (CVR) of the items was determined according to the evaluations obtained from field experts. Accordingly, CVR of the items varies between .33 and .86. In order to test the significance of the CVR, a table was drawn up by Veneziano and Hooper (1997) according to $\alpha=.05$ significance level. In this table, in order for each item to be included in the scale, CVR should be a minimum value of .49 .1 item $(\mathrm{CVR}=.33)$, 
which was below .49 in the obtained data, was removed from the form. According to the opinions and suggestions received from the experts, 4 items were added to the draft form and necessary revisions were done in terms of language expression and content. As a result, the 24-item draft DEGDSS was created.

At the implementation stage, the 24-item draft DEGDSS was made available to collect online data using the Google Forms with an added information text. Then, the draft scale form was shared in related social media groups and pages. The draft scale was filled out by 438 participants. However, some data removed due to missing information and outliers. EFA was conducted with the data of 218 participants and CFA with the data of 200 participants.

Within the scope of the validity and reliability studies of the DEGDSS, all EFA processes, item analysis and reliability calculated by using the SPSS 21 program. Firstly, Kaiser-Meyer-Olkin (KMO) and Bartlett Sphericity Test were conducted before EFA. After, the number of scale factors and the rotation method were determined and calculated. Results of the distribution of items to factors and rotated factor loading were presented. Next, CFA was conducted with 200 data by using LISREL 8.7 program. Results of the model according to good fit and acceptable fit values were compared. Then, item analysis was calculated in order to demonstrate a significant difference between the upper $27 \%$ and the lower $27 \%$ in terms of the total scores. Lastly, Cronbach Alpha value and correlations between the factors were calculated in order to present the internal consistency of the scale. The analysis conducted within the scope of the validity and reliability studies of DEGDSS are given in detail in the findings section. 


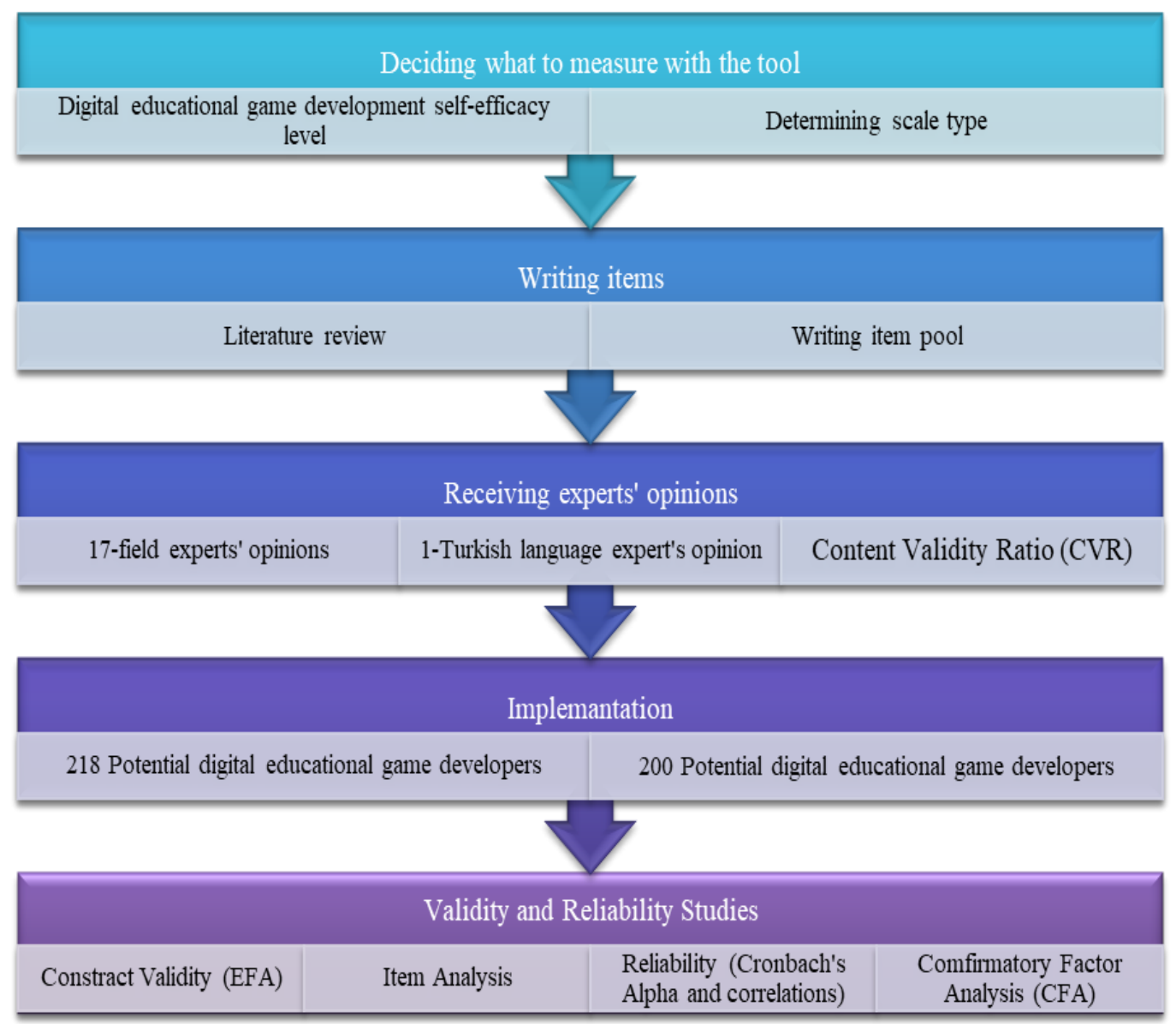

Figure 1. The steps of the DEGDSS development process

\section{Findings}

In this section, the validity and reliability studies of the Digital Game Development Self-Efficacy Scale were presented.

\section{Exploratory Factor Analysis}

EFA was performed with 24 items. KMO and Bartlett Sphericity Test were performed and the KMO value was calculated as $.965(\mathrm{p}=.000)$ to test whether the data obtained were suitable for EFA. According to Can (2014), the KMO value is higher than .70 and if it is meaningful, it means that there are relationships between items and the sample size is good for analysis. According to this result, the data are suitable for EFA.

EFA was performed without rotating to decide the factor number of the scale. As a result of the analysis, it was observed that there were two factors with eigenvalues greater than one. In factor analysis, the eigenvalue of a sub-dimension is expected to be 
at least 1\% (Aksu, Eser, \& Güzeller, 2017; Seçer, 2015). This situation was confirmed by examining the scree plot presented in Figure 2.

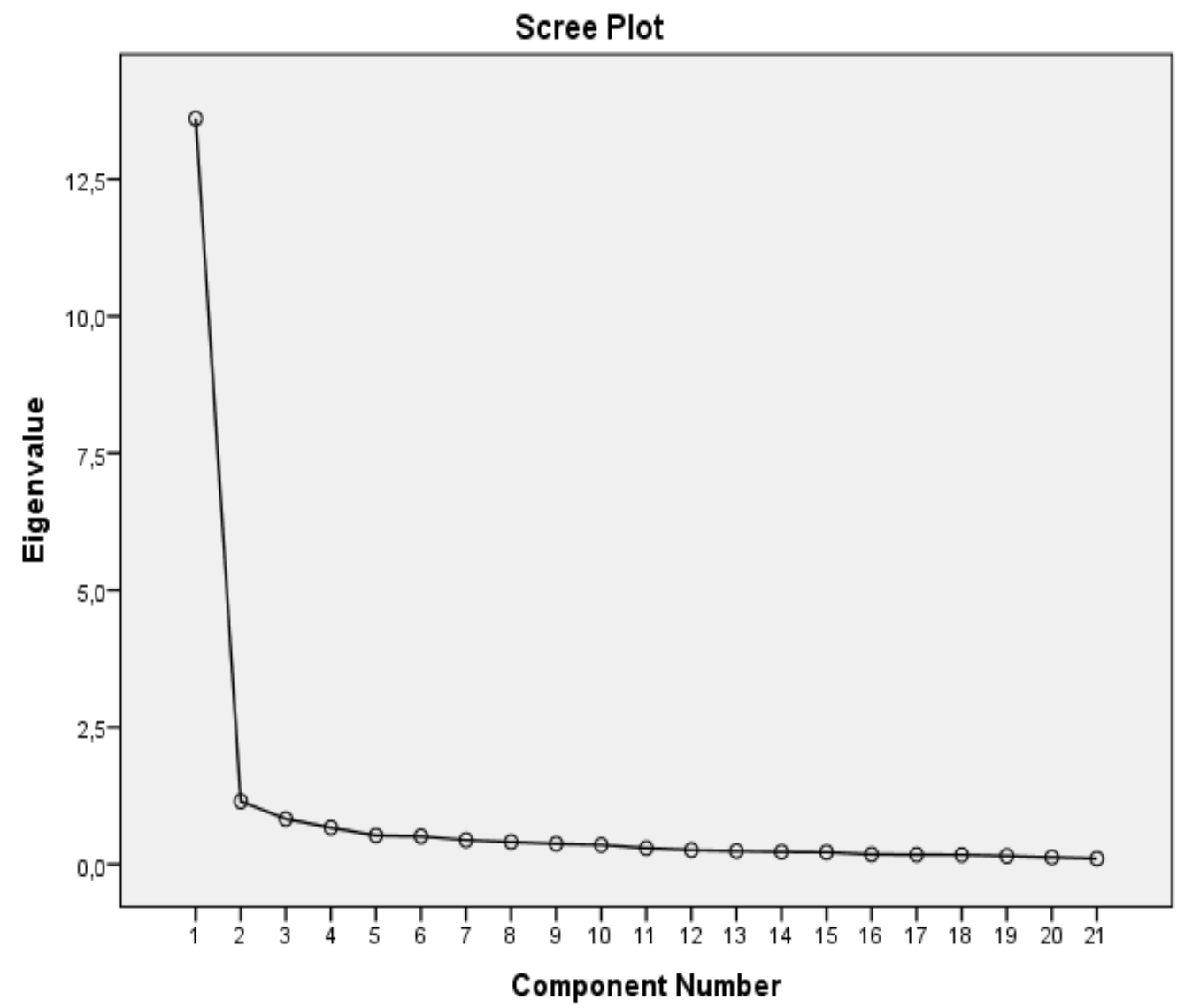

Figure 2. Scree plot of DEGDSS

Since the factors are considered to be independent of each other, the varimax rotation method is preferred (Ho, 2006). Varimax, one of the orthogonal rotation methods, is preferred because it eliminates the correlation between the factors and reveals the factors more clearly (Büyüköztürk, 2011; Can, 2014). As a result of the rotation, it is necessary to remove the items from the scale when the item loads are less than .30 and the correlations of the items with the factors is less than .10 (overlapping) (Çokluk, Şekercioğlu, \& Büyüköztürk, 2012). There was no item that has a low factor load of less than .30. However, 3 items (6., 7. and 13. items) were removed from the scale due to the overlapping. Based on these revisions, EFA was repeated. Distribution of items to factors and rotated factor loading is given in Table 2. 
Table 2. Distribution of items to factors and rotated factor loading

Rotated Factor

Loading

\begin{tabular}{|c|c|c|c|c|c|c|}
\hline \multirow[b]{2}{*}{ 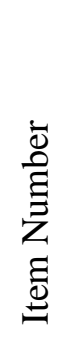 } & \multirow[b]{2}{*}{ Items } & & & \multicolumn{3}{|c|}{ Loading } \\
\hline & & 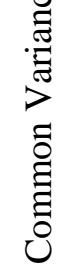 & 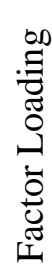 & ڤ్షేّ & 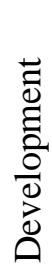 & 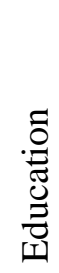 \\
\hline
\end{tabular}

S15

I can design educational game scenes in accordance with the visual design rules.

$.827 \quad .878 \quad .861$

S13

I can arrange an existing educational digital game scene design according to the visual design rules.

While creating an educational game design, I can use the visual design rules.

I can count the tools that can be used to develop an educational game.

I can list the visual design rules that should be present in an educational digital game.

I can choose the appropriate tools to develop an educational digital game.

I can evaluate educational digital game scene designs according to the visual design rules.

S14 I can prepare storyboards for educational game scenes.

Considering the technical capabilities of the tools, I can predict what features the educational game I will develop. educational digital game. 
When I write an educational digital game scenario, I can use the game elements appropriately.

I can use at least one tool to develop educational digital games.

I can complete an incomplete educational digital game scenario.

I can write an educational digital game scenario related to the existing learning outcome.

I can list the writing stages of an educational digital game scenario.

When developing an educational digital game, I can use one of the educational digital game design models.

I can test the usefulness of the developed educational digital game according to the appropriate techniques.

I can write learning outcomes in line with the needs of the target group to design an educational digital game.

I can determine the needs of the target group to design an educational digital game.

I can use the educational digital game elements in accordance with the existing learning outcome. 
After subtracting 3 items from the scale according to Table 2, 21 items that explain $70.25 \%$ of the total variance were obtained. The first factor explains $46.35 \%$ of the total variance of the scale and the second factor explains $23.9 \%$. The total variance value between $40 \%$ and $60 \%$ is claimed to be sufficient in the social sciences and at least $5 \%$ of the total variance announced for a factor to be relevant must be related to that factor (Çokluk, Şekercioğlu, \& Büyüköztürk, 2010; Tavşancıl, 2002). Rotated factor loadings vary between .602 and .861 . If the factor load value of items is .45 or higher, it means that the item measures the relevant factor well (Büyüköztürk, 2011). Based on this, it can be said that the relationship of the items in DEGDSS with the factors is at a very good level.

The remaining items consist of two factors as seen in the scree plot given in Figure 2. The first factor of the scale is called Game Development which is included 17-item, and the second factor is called Education which is included 4-item.

Whether the variable to be measured with the measurement tool differs according to the groups can be considered as evidence of the construct validity (Baykul, 2000). Therefore, it was examined whether the participants' DEGDSS scores differ from according to their attending to DEGD course and their experience in digital educational game development. Also, Cohen's $d$ was calculated to see the effect size of the differences. Cohen $d$ value vary between -1 and +1 , however, it can be greater than 1 (Riazi, 2016). Also, effect size is classified as 0.2 small-effect, 0.5 medium-effect, and 0.8 large-effect (Cohen, 1988). Table 3 shows the differences that participants' DEGDS according to their attending or not DEGD course. 
Table 3. Game developers' DEGD self efficacy levels according to their attending digital educational game development course

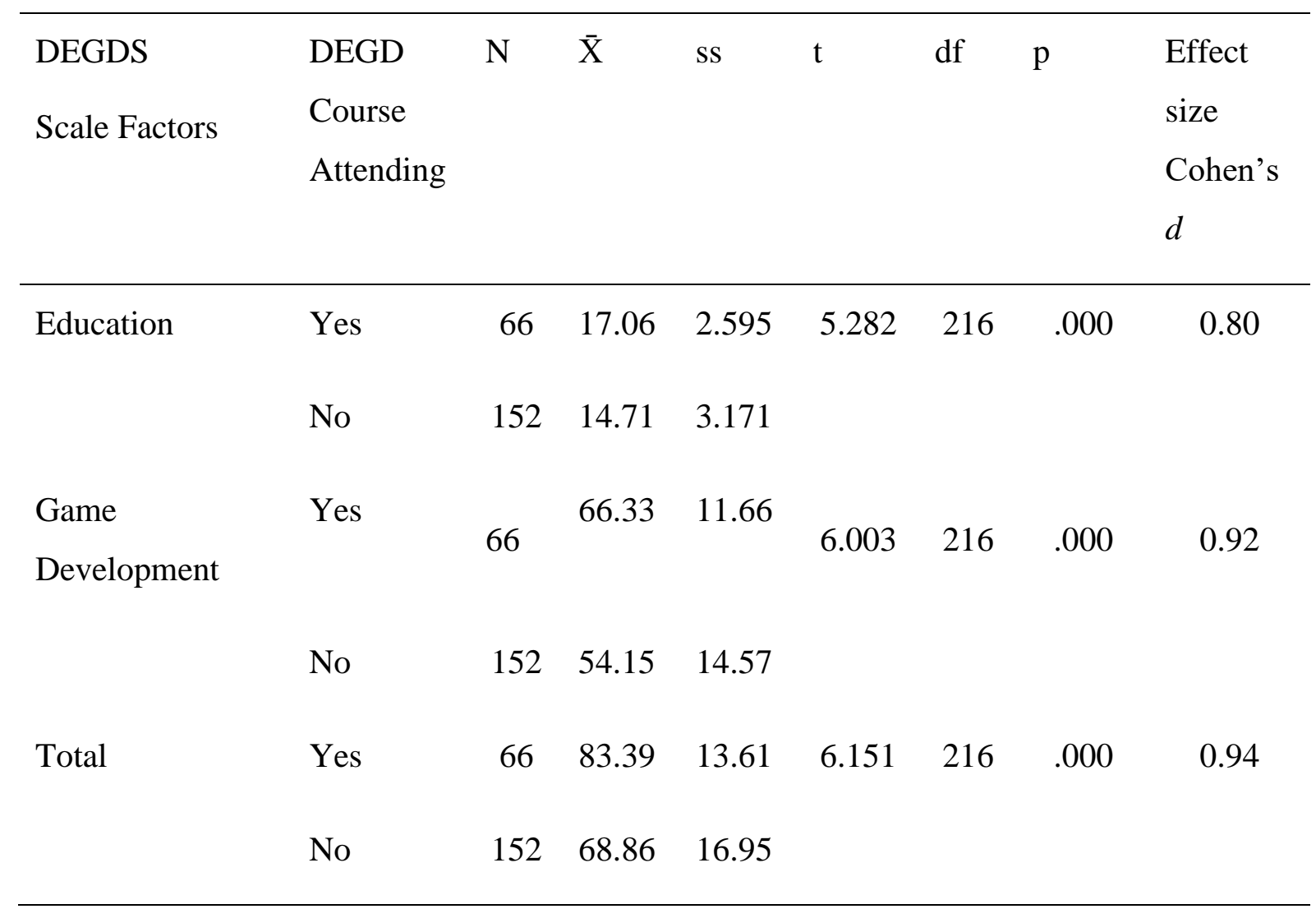

Independent group t-test was used to examine whether DEGDS has changed according to the status of game developers taking a digital educational game course. Results of this analysis, a significant difference was found in favor of who attended course according to DEGDS scores of the game developers both from the total of the scale and from the subscales of the scale $(\mathrm{p}<.05)$. Meaningful differences have a large effect size for a total of the scale and the two subscales $(d>0.8)$. Table 4 provides independent group t-test results to determine whether the participants' DEGDS levels differ according to their digital educational game development experience. 
Table 4. Game developers' DEGD self efficacy levels according to development of digital educational game

\begin{tabular}{lllllllll}
\hline DEGDS & DEGD & $\mathrm{N}$ & $\overline{\mathrm{X}}$ & $\mathrm{ss}$ & $\mathrm{t}$ & $\mathrm{df}$ & $\mathrm{p}$ & $\begin{array}{l}\text { Effect size } \\
\text { Cohen's d }\end{array}$ \\
Scale Factors & Development & & & & & & & \\
\hline Education & Yes & 78 & 17.01 & 2.371 & 6.428 & 216 & .000 & 0.86 \\
& No & 140 & 14.54 & 3.252 & & & & \\
Game & Yes & 78 & 66.30 & 10.572 & & & & \\
Development & & & & & 7.616 & 216 & .000 & 1.02 \\
& No & 140 & 53.12 & 14.799 & & & & \\
Total & Yes & 78 & 83.32 & 12.277 & 7.777 & 216 & .000 & 1.43 \\
& No & 140 & 67.66 & 17.229 & & & &
\end{tabular}

When DEGDS was evaluated by independent group t-test according to the participants' experience of game development, it was found that those with game development experience were significantly higher than those without experience $(p<05)$. Meaningful differences for a total of the scale and two subscales have a large affect $(d>0.8)$.

\section{Item Analysis}

In order to perform item analysis for the DEGSS, the raw scores obtained from the scale are listed as small to large. Afterward, groups were formed with 118 participants with the highest and lowest scores to determine the lower $27 \%$ and upper $27 \%$. According to the results of the analysis, it was observed that there was a statistically significant difference between the upper $27 \%$ and the lower $27 \%$ in terms of the total scores obtained from the DEGDSS $(\mathrm{t}(116)=26.624 ; \mathrm{p}=.000)$. However, for each item of the scale, the $t$ value of the difference between the upper and the lower group mean scores are calculated and presented in Table 5. In addition, item-total correlations are between .567 and .862 . These values show that some of the items have medium $(.30-.70)$ and some of them have high (.70 - 1.00) item test correlations (Büyüköztürk, 2011). 
Table 5. Independent t-test results for independent groups for sub-27\% and upper $27 \%$ of the scale

\begin{tabular}{|c|c|c|c|c|c|c|c|}
\hline $\begin{array}{l}\text { Item } \\
\text { No }\end{array}$ & Group & $\mathrm{N}$ & $\bar{X}$ & SS & df & $\mathrm{t}$ & $\mathrm{p}$ \\
\hline \multirow{2}{*}{ s1 } & Upper group & 59 & 4.81 & .472 & \multirow{2}{*}{116} & \multirow{2}{*}{13.355} & \multirow{2}{*}{.000} \\
\hline & Subgroup & 59 & 3.11 & .852 & & & \\
\hline \multirow{2}{*}{$\mathrm{s} 2$} & Upper group & 59 & 4.69 & .701 & \multirow{2}{*}{116} & \multirow{2}{*}{10.670} & \multirow{2}{*}{.000} \\
\hline & Subgroup & 59 & 3.11 & .892 & & & \\
\hline \multirow{2}{*}{ s3 } & Upper group & 59 & 4.66 & .575 & \multirow{2}{*}{116} & \multirow{2}{*}{11.318} & \multirow{2}{*}{.000} \\
\hline & Subgroup & 59 & 3.01 & .955 & & & \\
\hline \multirow{2}{*}{ s4 } & Upper group & 59 & 4.40 & .790 & \multirow{2}{*}{116} & \multirow{2}{*}{9.018} & \multirow{2}{*}{.000} \\
\hline & Subgroup & 59 & 2.83 & 1.082 & & & \\
\hline \multirow{2}{*}{ s5 } & Upper group & 59 & 4.54 & .702 & \multirow{2}{*}{116} & \multirow{2}{*}{14.055} & \multirow{2}{*}{.000} \\
\hline & Subgroup & 59 & 2.54 & .837 & & & \\
\hline \multirow{2}{*}{ s6 } & Upper group & 59 & 4.66 & .512 & \multirow{2}{*}{116} & \multirow{2}{*}{14.108} & \multirow{2}{*}{.000} \\
\hline & Subgroup & 59 & 2.71 & .929 & & & \\
\hline \multirow{2}{*}{ s7 } & Upper group & 59 & 4.71 & .493 & \multirow{2}{*}{116} & \multirow{2}{*}{15.431} & \multirow{2}{*}{.000} \\
\hline & Subgroup & 59 & 2.49 & .989 & & & \\
\hline \multirow{2}{*}{ s8 } & Upper group & 59 & 4.77 & .418 & \multirow{2}{*}{116} & \multirow{2}{*}{17.369} & \multirow{2}{*}{.000} \\
\hline & Subgroup & 59 & 2.54 & .896 & & & \\
\hline \multirow{2}{*}{ s9 } & Upper group & 59 & 4.72 & .448 & \multirow{2}{*}{116} & \multirow{2}{*}{16.815} & \\
\hline & Subgroup & 59 & 2.62 & .848 & & & .000 \\
\hline & Upper group & 59 & 4.71 & .456 & & & \\
\hline SIO & Subgroup & 59 & 2.64 & .942 & 116 & 15.164 & .000 \\
\hline
\end{tabular}




\begin{tabular}{|c|c|c|c|c|c|c|c|}
\hline \multirow{2}{*}{ s11 } & Upper group & 59 & 4.71 & .493 & \multirow{2}{*}{116} & \multirow{2}{*}{17.848} & \multirow{2}{*}{.000} \\
\hline & Subgroup & 59 & 2.35 & .885 & & & \\
\hline \multirow{2}{*}{ s 12} & Upper group & 59 & 4.66 & .544 & \multirow{2}{*}{116} & \multirow{2}{*}{17.832} & \multirow{2}{*}{.000} \\
\hline & Subgroup & 59 & 2.30 & .856 & & & \\
\hline \multirow{2}{*}{ s13 } & Upper group & 59 & 4.72 & .448 & \multirow{2}{*}{116} & \multirow{2}{*}{19.409} & \multirow{2}{*}{.000} \\
\hline & Subgroup & 59 & 2.38 & .809 & & & \\
\hline \multirow{2}{*}{ s14 } & Upper group & 59 & 4.62 & .613 & \multirow{2}{*}{116} & \multirow[t]{2}{*}{15.807} & \multirow{2}{*}{.000} \\
\hline & Subgroup & 59 & 2.37 & .907 & & & \\
\hline \multirow{2}{*}{ s 15} & Upper group & 59 & 4.76 & .429 & \multirow{2}{*}{116} & \multirow{2}{*}{19.486} & \multirow{2}{*}{.000} \\
\hline & Subgroup & 59 & 2.35 & .846 & & & \\
\hline \multirow{2}{*}{ s16 } & Upper group & 59 & 4.72 & .448 & \multirow{2}{*}{116} & \multirow{2}{*}{15.950} & \multirow{2}{*}{.000} \\
\hline & Subgroup & 59 & 2.54 & .952 & & & \\
\hline \multirow{2}{*}{ s17 } & Upper group & 59 & 4.62 & .553 & \multirow{2}{*}{116} & \multirow{2}{*}{18.498} & \multirow{2}{*}{.000} \\
\hline & Subgroup & 59 & 2.22 & .831 & & & \\
\hline \multirow{2}{*}{ s18 } & Upper group & 59 & 4.76 & .429 & \multirow{2}{*}{116} & \multirow{2}{*}{19.361} & \multirow{2}{*}{.000} \\
\hline & Subgroup & 59 & 2.44 & .815 & & & \\
\hline \multirow{2}{*}{ s19 } & Upper group & 59 & 4.74 & .476 & \multirow{2}{*}{116} & & \\
\hline & Subgroup & 59 & 2.42 & .855 & & & \\
\hline & Upper group & 59 & 4,77 & .617 & & 16.756 & \\
\hline & Subgroup & 59 & 2,44 & .876 & & & \\
\hline & Upper group & 59 & 4,64 & .580 & & & \\
\hline & Subgroup & 59 & 2,49 & .935 & & & \\
\hline
\end{tabular}




\section{Reliability}

Internal consistency coefficients were calculated within the scope of the reliability study of DEGDSS. The Cronbach Alpha coefficient for the first factor which is Education was .836 , for the second factor which is Game Development is .973 and for the total of the scale was .972 . Since the reliability coefficients of .70 and above are considered to be reliable (Büyüköztürk, 2011; Fraenkel, Wallen, \& Hyun, 2012), it can be said that the calculated reliability coefficients are sufficient. Furthermore, there is a significant relationship between game development and education factors $(\mathrm{r}=.739, \mathrm{p}=<.00 ; \mathrm{N}=$ 218). In this respect, it shows an acceptable level of reliability in dimensions.

\section{Confirmatory Factor Analysis}

CFA was performed to test the validation of the factor structure associated with EFA. In order to determine the suitability of the model, the values recommended by Schermelleh-Engel, Moosbrugger, \& Müller (2003) which are $\chi^{2} /$ df, Root Mean Square Error of Approximation (RMSEA), Standardized Root Meaning (SRMR), Normed Fit Index (NFI), Comparative Fit Index (CFI), Goodness of Fit Index (GFI) and Adjusted Goodness of Fit Index (AGFI) are taken as reference. The results of the model according to good fit and acceptable fit values are given in Table 6.

In comparison with the acceptable and perfect fit index values of the model seen in Table 6 and the fit index values obtained from CFA, $\chi^{2} / \mathrm{df}=2.047(\mathrm{p}=<0.01)$ was determined. RMSEA $=.073 ;$ SRMR $=.039 ; \mathrm{NFI}=.97, \mathrm{NNFI}=.98, \mathrm{CFI}=.98$; GFI $=.84$ and AGFI $=.80$. The results obtained are acceptable even if they do not have excellent fit values. These findings confirm the factor structure of DEGDSS as seen in Figure 3. 
Table 6. Confirmatory factor analysis results

\begin{tabular}{lllc}
\hline Fit Measure & Good Fit & Acceptable Fit & Suggested Fit Measures \\
\hline$\chi 2$ df & $0 \leq \chi 2 / \mathrm{df} \leq 2$ & $2<\chi 2 / \mathrm{df} \leq 3$ & 2.047 \\
RMSEA & $0 \leq \mathrm{RMSEA} \leq .05$ & $05<\mathrm{RMSEA} \leq .08$ & 0.073 \\
SRMR & $0 \leq \mathrm{SRMR} \leq .05$ & $.05<\mathrm{SRMR} \leq .10$ & 0.039 \\
NFI & $.95 \leq \mathrm{NFI} \leq 1.00$ & $.90 \leq \mathrm{NFI}<.95$ & 0.97 \\
NNFI & $.97 \leq \mathrm{NNFI} \leq 1.00$ & $.95 \leq \mathrm{NNFI}<.97$ & 0.98 \\
CFI & $.97 \leq \mathrm{CFI} \leq 1.00$ & $95 \leq \mathrm{CFI}<.97$ & 0.98 \\
GFI & $.95 \leq \mathrm{GFI} \leq 1.00$ & $.90 \leq \mathrm{GFI}<.95$ & 0.84 \\
AGFI & $.90 \leq \mathrm{AGFI} \leq 1.00$ & $.85 \leq \mathrm{AGFI}<.90$ & 0.80 \\
\hline
\end{tabular}




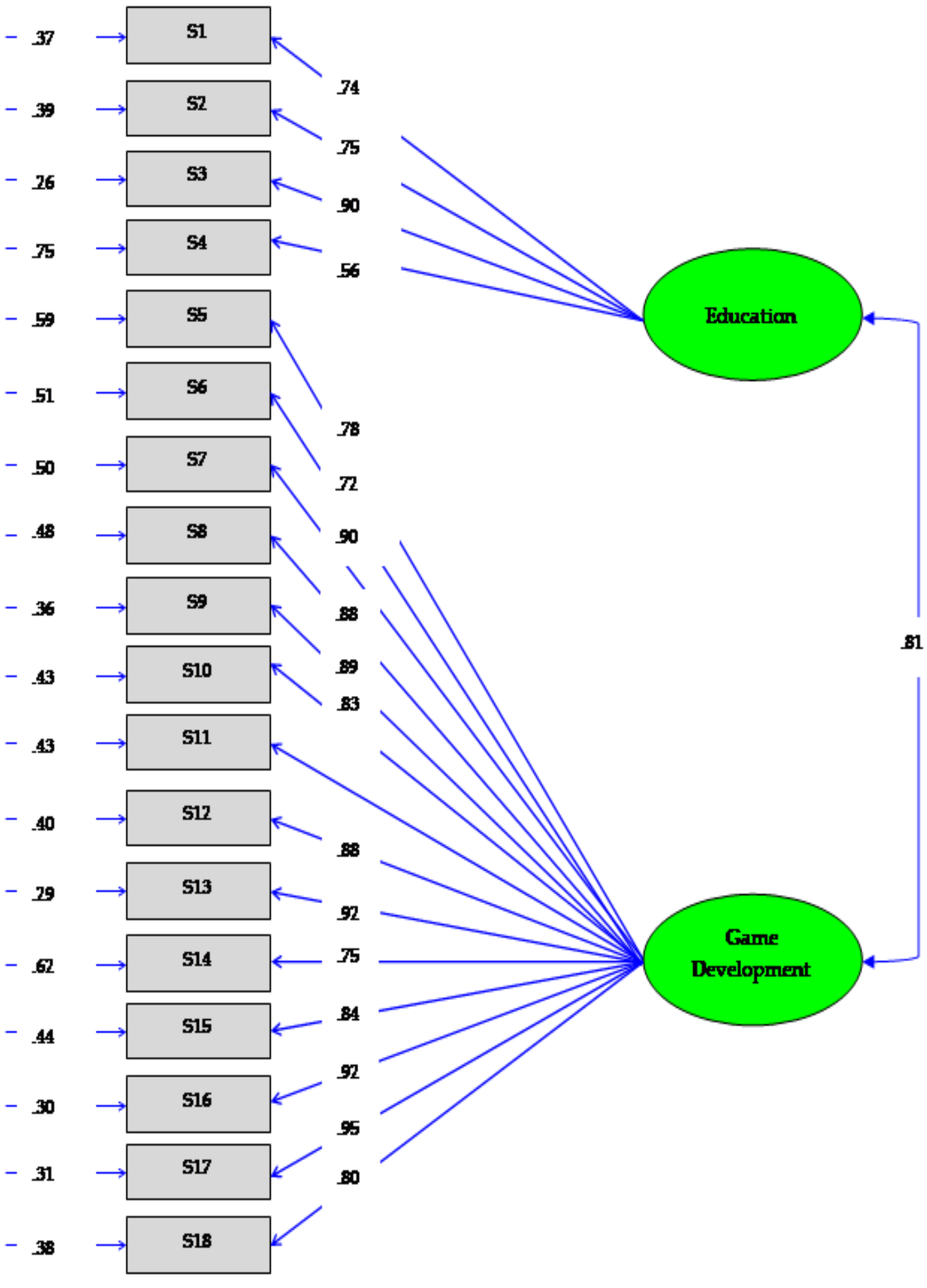

Chi-Square $=376.77, \mathrm{df}=184, p=0.00000, \mathrm{RMSEA}=0.073$

Figure 3. Confirmatory factor analysis estimated numbers 


\section{Discussion and Results}

In this study, it is aimed to develop valid and reliable Digital Educational Game Development Self-Efficacy Scale. And it is determined that game developers' DEGDS levels according to their attending DEGD course and their experience in DEGD.

The validity and reliability analysis results obtained from this study reveal that DEGDSS can be used to measure the DEGDS levels of potential DEG developers. Also, based on results obtained from DEGDSS, it has been revealed that attending DEGD course and experiencing DEGD increases game developers' DEGDS levels. Bandura (1997) stated that one of the main factors affecting self-efficacy is direct experiences. In addition, the positive experiences and the receiving of a course can positively affect their self-efficacy (Tschannen-Moran \& Hoy, 2007). Findings obtained with DEGDSS support these studies.

DEGDSS consists of 21 items. Items of the DEGDSS are in a 5-degree Likert format. The status of participation in each item is graded as "I totally agree" (5), "I agree" (4), "I am undecided" (3), "I disagree" (2) and "I totally disagree" (1). DEGDSS is composed of two factors. The first factor of the scale is called Education which includes 4-item and the second factor is called Game Development which includes 17item. Minimum 21 points and a maximum of 105 points can be obtained from the scale. As the scores obtained from the scale increase, the individuals' DEGDS levels increase. DEGDSS is given in appendix.

For the effective development of DEGs, there are digital educational game development models, which include analysis, design, development, implementation, and evaluation phases, recommended in related studies (Akgün et al., 2011; Ak1llı \& Çağıltay, 2006; Zin et al., 2009). In addition to these phases, Kocadere et al. (2019) included preliminary analysis phase before the analysis phase.It can be said that the tasks that are desired to be carried out in the preliminary analysis/analysis phases of these models coincide with the Education dimension of DEGDSS. It can also be said that the content of DEGDSS in the game development dimension is similar to the tasks that are desired to be performed in the design, development, and application phases of digital educational game development models. The tasks to be carried out during the 
evaluation phase are both in the education and game development dimension of DEGDSS. As a matter of fact, in digital educational game development models, the evaluation phase takes place in the entire digital educational game development process. Accordingly, it can be said that DEGDSS is compatible with the model to be used when developing a digital educational game in general. Also, the related studies reveal that individuals with high self-efficacy level on a particular subject tend to perform better on that subject (Usher, Li, Butz, \& Rojas, 2019). When considered from this point of view, it can be stated that according to the results obtained from DEGDSS, digital educational game developers with high DEGDS self-efficacy level can develop more effective digital educational games. At the same time, the skills envisaged to be acquired within the scope of digital educational game development training (Kelleci \& Kulaksız, 2017; $2018 \mathrm{a} ; 2018 \mathrm{~b})$ are paralleled with the competencies specified in the articles of DEGDSS.

The number of qualified digital educational game developers should increase in order to enhance the quality of digital educational games. DEGD trainings/courses can increase the widespread effect of DEGDSS by enabling the training of potential DEG developers. Therefore, in future studies, it is recommended to apply to different characteristic groups of participants. It is suggested to investigate the factors affecting DEGDS by including different variables in the research process with DEGDSS and to use DEGDSS to test the effectiveness of DEGD courses/training. 


\section{Ethical Approval:}

Hasan Kalyoncu University, Social Sciences, and Humanities Institute of Research Ethics Committee (Date: 16.03.2020, No: 2003160012) provided ethics approval for this study.

\section{Conflict of Interest:}

Authors have no conflict of interest to declare.

\section{Author Contributions:}

The first author's contribution was that conceptualization of the research problem, literature review, data collection and analysis. The second author's contribution was that conceptualization of the research problem, design of the methodology, data collection and analysis. All authors contributed to the discussion section and reporting. 


\section{References}

Akgün, E., Nuhoğlu, P., Tüzün, H., Kaya, G., \& Çınar, M. (2011). Bir eğitsel oyun tasarımı modelinin geliştirilmesi [Development of an educational game design model]. Eğitim Teknolojisi Kuram ve Uygulama, 1(1), 41-61.

Akıllı, K. G., \& Çağıltay, K. (2006). An instructional design/development model for the creation of game-like learning environments the FIDGE model, In M. Pivec (Ed.), Affective and emotional aspects of human-computer interaction: Gamebased and innovative learning approaches (pp. 93-112). Amsterdam, Netherlands: IOS Press.

Aksu, G., Eser, M. T., \& Güzeller, C. O. (2017). Açımlayıcı ve doğrulayıcı faktör analizi ile yapısal eşitlik uygulamaları. Ankara: Detay Yayıncılık.

Aleem, S., Capretz, L. F., \& Ahmed, F. (2016). Critical success factors to improve the game development process from a developer's perspective. Journal of Computer Science and Technology, 31(5), 925-948.

An, Y. J., \& Cao, L. (2017). The effects of game design experience on teachers' attitudes and perceptions regarding the use of digital games in the classroom. TechTrends, 61(2), 162-170.

An, Y-J., Haynes, L, D'Alba, A., \& Chumney, F. (2016). Using educational computer games in the classroom: Science teachers' experiences, attitudes, perceptions, concerns, and support needs. Contemporary Issues in Technology \& Teacher Education, 16(4), 415-433.

Aşkar, P., \& Dönmez, O. (2004). Eğitim yazılımı geliştirme öz-yeterlik algısı ölçeği [A self-efficacy scale for educational software development]. Eğitim Bilimleri ve Uygulama, 3(6), 259-274.

Bakaç, E., \& Özen, R. (2015). Materyal tasarımı öz-yeterlik inancı ölçeği: Geçerlik ve güvenirlik çalışması [Material design self- efficacy belief scale: A validity and reliability study]. International Journal of Human Sciences, 12(2), 461-476.

Bandura, A. (1997). Self-efficacy: The exercise of self-control. New York: Freeman. 
Baykul, Y. (2000). Eğitimde ve psikolojide ölçme (3th Edition) [Measurement in education and psychology]. Ankara: Pegem Akademi.

Brom, C., Dechterenko, F., Sisler, V., Hlavka, Z., \& Lukavsky, J. (2018). Does motivation enhance knowledge acquisition in digital game-based and multimedia learning? A review of studies from one lab. In Göbel S. et al. (Eds.) Serious Games. JCSG 2018. Lecture Notes in Computer Science, vol 11243. Springer, Cham.

Brown, Q., Lee, F., \& Alejandre, S. (2009, April). Emphasizing soft skills and team development in an educational digital game design course. In Fourth International Conference on the Foundations of Digital Games, 240-247.

Bryman, A., \& Cramer, D. (1999). Quantitative data analysis with SPSS release 8 for Windows: A guide for social scientists. London: Routledge.

Büyüköztürk, Ş. (2011). Sosyal bilimler için veri analizi el kitabı (15th Edition) [Data analysis handbook for social sciences]. Ankara: Pegem Akademi.

Büyüköztürk, Ş., Çakmak, K. Ç., Akgün, E. Ö., Karadeniz, Ş., \& Demirel, F. (2015). Bilimsel araştırma yöntemleri (19th Edition) [Scientific research methods]. Ankara: Pegem Akademi.

Byun, J., \& Joung, E. (2018). Digital game-based learning for K-12 mathematics education: A meta-analysis. School Science and Mathematics. 118, 113-126.

Cai, L., Liu, F., \& Liang, Z. (2010, December). The research and application of education game design model in teaching Chinese as a Foreign Language. In 2010 IEEE International Conference on Progress in Informatics and Computing (Vol. 2, pp. 1241-1245). China: IEEE.

Can, A. (2014). SPSS ile bilimsel araştırma sürecinde nicel veri analizi. Ankara: Pegem Akademi.

Cherian, J., \& Jacob, J. (2013). Impact of self efficacy on motivation and performance of employees. International Journal of Business and Management, 8(14), 80-88. 
Chen, C-H., Shih, C.-C., \& Law, V. (2020). The effects of competition in Digital GameBased Learning (DGBL): A meta-analysis. Educational Technology Research and Development, 68(3), 1-19. doi: 10.1007/s11423-020-09794-1.

Cohen, J. (1988). Statistical power analysis for the behavioral sciences (2nd Edition). Hillsdale, NJ: Lawrence Erlbaum.

Cronbach, L. J. (1990). Essentials of psychological testing (5th Edition). New York: Harper-Row.

Çokluk, Ö., Şekercioğlu, G., \& Büyüköztürk, Ş. (2010). Sosyal bilimler için çok değişkenli istatistik. Ankara: Pegem Yayıncılık.

Çokluk, Ö., Şekercioğlu, G., \& Büyüköztürk, Ş. (2012). Sosyal bilimler için çok değişkenli SPSS ve LISREL uygulamaları. Ankara: Pegem A Yayıncılık.

DeVellis, R. F. (2003). Scale development: Theory and application (6th Edition). Thousand Oaks, CA: SAGE.

Fraenkel, J. R., Wallen, N. E., \& Hyun, H. H. (2012). How to design and evaluate research in education (8th Edition). New York, NY: McGraw Hill Education.

Gaudelli, W., \& Talyor, A. (2011). Modding the global classroom? Serious video games and teacher reflection. Contemporary Issues in Technology and Teacher Education, 11(1), 70-91.

Gros, B. (2015). Integration of digital games in learning and e-learning environments: Connecting experiences and context. In Digital Games and Mathematics Learning (pp. 35-53). Springer: Dordrecht.

Gürbüz, S., \& Şahin, F. (2018). Sosyal bilimlerde araştırma yöntemleri [Research methods in social sciences] (5th Edition). Ankara: Seçkin Yayıncılık.

Hava, K., \& Cakir, H. (2017). A systematic review of literature on students as educational computer game designers. In J. Johnston (Ed.), Proceedings of EdMedia 2017 (pp. 407-419). Washington, DC: Association for the Advancement of Computing in Education. 
Hewner, M., \& Guzdial, M. (2010, March). What game developers look for in a new graduate: Interviews and surveys at one game company. 41st ACM Technical Symposium on Computer Science Education, Milwaukee, Wisconsin, USA.

Ho, R. (2006). Handbook of univariate and multivariate data analysis and interpretation with SPSS. New York: Taylor and Francis.

International Society for Technology in Education. (2017). ISTE standards for educators. Retrieved from http://www.iste.org/standards/for-educators.

Kelleci, Ö., \& Kulaksız, T. (2017, October). Eğitsel Oyun Tasarlama Eğitiminin etkililiğinin değerlendirilmesi. 5. International Instructional Technologies and Teacher Education Symposium, Izmir, Turkey.

Kelleci, Ö., \& Kulaksız, T. (2018a, May). Bir dijital eğitsel oyun tasarlama eğitiminin performansa dayalı olarak karşılaştırmalı değerlendirilmesi: Öğretmen adayı perspektifi. 12th International Computer \& Instructional Technologies Symposium (ICITS 2018), Izmir, Turkey.

Kelleci, Ö., \& Kulaksız, T. (2018b, April). Developing an educational digital game design course. 8. World Conference On Educational Technology Researches, Batum, Georgia.

Kocadere, S. A., Özhan, Ş. Ç., Bayrak, F., \& Kibar, P. N. (2019). Herkül'ün hikayesi: Bir eğitsel oyun modeli önerisi. Eğitim Teknolojisi Kuram ve Uygulama, 9(1), 230-250. doi: 10.17943/etku.451990.

Law, E. L., \& Sun, X. (2012). Evaluating user experience of adaptive digital educational games with activity theory. International Journal of Human Computer Studies, 70(7), 478-497. doi: 10.1016/j.ijhcs.2012.01.007.

Li, Q., Lemieux, C., Vandermeiden, E., \& Nathoo, S. (2013). Are you ready to teach secondary mathematics in the 21 st century? A study of preservice teachers' digital game design experience. Journal of Research on Technology in Education, 45(4), 309-337. doi: 10.1080/15391523.2013.10782608.

Martins, A. R., \& Oliveira, L. R. (2018, June). Students as Creators of Educational Games-Learning to Use Simple Frameworks and Tools to Empower Students as 
Educational Game Designers. In EdMedia+ Innovate Learning (pp. 1210-1215). Association for the Advancement of Computing in Education (AACE).

McGill, M. M. (2008, November). Critical skills for game developers: An analysis of skills sought by industry. 2008 Conference on Future Play: Research, Play, Share. Toronto, Canada.

McMahon, M. T. (2009). Using the DODDEL model to teach serious game design to novice designers. Proceedings of Ascilite 2009 (pp. 646-653). Auckland, New Zealand. The University of Auckland, Auckland University of Technology \& Australasian Society for Computers in Learning in Tertiary Education.

Ministry of National Education. (2020). Öğretmenlik mesleği genel yeterlikleri. Retrieved from http://oygm.meb.gov.tr/www/ogretmenlik-meslegi-genelyeterlikleri/icerik/39.

Noemi, P., \& Maximo, S. H. (2014). Educational games for learning. Universal Journal of Educational Research, 2(3), 230-238. doi: 10.13189/ujer.2014.020305.

Prensky, M. (2001). Digital game-based learning. New York: McGraw Hill.

Prensky, M. (2008). Students as designers and creators of educational computer games: Who else? British Journal of Educational Technology, 39(6), 1004-1019. doi:10.1111/j.1467-8535.2008.00823_2.x

Ramalingam, V., \& Wiedenbeck, S. (1998). Development and validation of scores on a computer programming self-efficacy scale and group analyses of novice programmer self-efficacy. Journal of Educational Computing Research, 19(4), 367-381. doi: 10.2190/c670-y3c8-1tj1-ct3p

Riazi, A. M. (2016). The Routledge encyclopedia of research methods in applied linguistics. NY: Routledge.

Sancar Tokmak, H., \& Özgelen, S. (2013). The ECE pre-service teachers' perception on factors affecting the integration of educational computer games in two conditions: Selecting versus redesigning. Educational Sciences: Theory and Practice, 13(2) - Spring, 1345-1356. 
Schermelleh-Engel, K., Moosbrugger, H., \& Müller, H. (2003). Evaluating the fit of structural equation models: Tests of significance and descriptive goodness-of-fit measures. Methods of Psychological Research Online, 8(2), 23-74.

Seçer, İ. (2015). Psikolojik test geliştirme ve uyarlama süreci. Ankara: Anı Yayıncılık.

Talan, T., Doğan, Y., \& Batd1, V. (2020). Efficiency of digital and nondigital educational games: A comparative meta-analysis and a meta-thematic analysis. Journal of Research on Technology in Education, 52(4), 474-514. doi:10.1080/15391523.2020.1743798.

Tavşancıl, E. (2002). Tutumların ölçülmesi ve spss ile veri analizi. Ankara: Nobel Yayıncilık.

Tschannen-Moran, M., \& Hoy, A. W. (2007). The differential antecedents of selfefficacy beliefs of novice and experienced teachers. Teaching and Teacher Education, 23(6), 944-956. doi:10.1016/j.tate.2006.05.003

Tüzün, H., Barab, S. A., \& Thomas, M. K. (2019). Reconsidering the motivation of learners in educational computer game contexts. Turkish Journal of Education, 8(2), 129-159. doi: 10.19128/turje.546283

Usher, E. L., Li, C. R., Butz, A. R., \& Rojas, J. P. (2019). Perseverant grit and selfefficacy: Are both essential for children's academic success? Journal of Educational Psychology, 111(5), 877-902. doi:10.1037/edu0000324.

Veneziano L., \& Hooper J. (1997). A method for quantifying content validity of healthrelated questionnaires. American Journal of Health Behavior, 21(1), 67-70.

Worthington, R. W., \& Whittaker, T. A. (2006). Scale development research: A content analysis and recommendations for best practices. The Counseling Psychologist, 34(6), 806-838. doi:10.1177/0011000006288127.

Yang, Y. T. C. (2015). Virtual CEOs: A blended approach to digital gaming for enhancing higher order thinking and academic achievement among vocational high school students. Computers \& Education, 81, 281-295. doi:10.1016/j.compedu.2014.10.004. 
Yien, J. M., Hung, C. M., Hwang, G. J., \& Lin, Y. C. (2011). A game-based learning approach to improving students' learning achievements in a nutrition course. Turkish Online Journal of Educational Technology, 10(2), 1-10.

Zin, N. A., Jaafar, A., \& Yue, W. S. (2009). Digital Game-Based Learning (DGBL) model and development methodology for teaching history. WSEAS Transactions on Computers, 8(2), 322-333. 